\title{
Exact SU(5) Yukawa matrix unification in the general flavour violating MSSM
}

\author{
Mateusz Iskrzyński ${ }^{a}$ and Kamila Kowalska ${ }^{b}$ \\ ${ }^{a}$ Institute of Theoretical Physics, University of Warsaw, \\ Pasteura 5, 02-093 Warsaw, Poland \\ ${ }^{b}$ National Centre for Nuclear Research, \\ Hoża 69, 00-681 Warsaw, Poland \\ E-mail: mateusz.iskrzynski@fuw.edu.pl, kamila.kowalska@fuw.edu.pl
}

ABSTRACT: We investigate the possibility of satisfying the SU(5) boundary condition $\mathbf{Y}^{d}=\mathbf{Y}^{e T}$ at the GUT scale within the renormalizable $R$-parity conserving Minimal Supersymmetric Standard Model (MSSM). Working in the super-CKM basis, we consider non-zero flavour off-diagonal entries in the soft SUSY-breaking mass matrices and the $A$ terms. At the same time, the diagonal $A$-terms are assumed to be suppressed by the respective Yukawa couplings. We show that a non-trivial flavour structure of the soft SUSY-breaking sector can contribute to achieving precise Yukawa coupling unification for all three families, and that the relevant flavour-violating parameters are $\left(m_{\tilde{d}}^{2}\right)_{23},\left(m_{\tilde{d}}^{2}\right)_{12}$, $\left(m_{\tilde{d}}^{2}\right)_{13}$ and $A_{12 / 21}^{d}$. We indicate the parameter space regions where the Yukawa unification condition can be satisfied, and we demonstrate that it is consistent with a wide set of experimental constraints, including flavour and electroweak observables, Higgs physics and the LHC bounds. However, as a consequence of the down-electron Yukawa unification requirement, the MSSM vacuum in our scenario is metastable, though long-lived. We also point out that the lightest neutralino needs to be almost purely bino-like and relatively light, with the mass in the ballpark of $250 \mathrm{GeV}$. Since the proper value of the dark matter relic density is in this case obtained through co-annihilation with a sneutrino, at least one generation of sleptons must be light. Such a clear experimental prediction makes the flavour-violating SU(5) Yukawa unification scenario fully testable at the LHC $\sqrt{s}=14 \mathrm{TeV}$ with the 3-lepton searches for electroweakino production.

KeYwords: Supersymmetry Phenomenology

ARXiv EPRINT: 1412.8651 


\section{Contents}

1 Introduction 1

2 Anatomy of the minimal SU(5) Yukawa unification 3

2.1 Flavour-violating threshold corrections to the Yukawa matrices at the decoupling scale

3 Impact of the GFV threshold corrections on Yukawa unification

3.1 Numerical tools and scanning methodology

3.2 Input SUSY parameters

$\begin{array}{lll}3.3 & \text { Yukawa unification through flavour-violating terms } & 10\end{array}$

4 Phenomenology of the unification scenario 13

4.1 Experimental constraints 13

$\begin{array}{lll}\text { 4.1.1 Dark matter } & 14\end{array}$

$\begin{array}{lll}\text { 4.1.2 Higgs, flavour and electroweak observables } & 17\end{array}$

$\begin{array}{lll}4.1 .3 & \text { LHC direct SUSY searches } & 19\end{array}$

$\begin{array}{lll}4.2 & \text { EW vacuum stability } & 21\end{array}$

4.3 Examples of points with successful SU(5) Yukawa matrix unification 22

5 Conclusions

\section{Introduction}

One of the virtues of supersymmetry (SUSY) is that it allows unification of the gauge couplings. Such a feature suggests that above the unification scale the Minimal Supersymmetric Standard Model (MSSM) should be replaced with a more general theory. The simplest realization of a Grand Unified Theory (GUT) is based on the SU(5) gauge symmetry [1], and its straightforward consequence is the equality of the Yukawa couplings of down-type quarks and charged leptons at the GUT scale. Given the experimentally measured values of the fermion masses, such a condition is easy to satisfy for the third family of fermions. On the other hand, obtaining $Y_{s}\left(M_{\mathrm{GUT}}\right)=Y_{\mu}\left(M_{\mathrm{GUT}}\right)$ and $Y_{d}\left(M_{\mathrm{GUT}}\right)=Y_{e}\left(M_{\mathrm{GUT}}\right)$ turns out to be quite a non-trivial task in the MSSM. Several solutions to this problem have been proposed in the literature, which either considered an extended Higgs sector above the GUT scale [2], or employed higher-dimensional operators [3-5].

Another approach to Yukawa coupling unification is based on the observation that SUSY threshold corrections at the superpartner decoupling scale can considerably alter or even generate the masses of light fermions [6]. Such a possibility was first investigated in the context of SUSY grand unification in ref. [7], where the presence of general flavourviolating interactions in the soft SUSY-breaking Lagrangian was assumed. More recently, 
several studies have been devoted to the possibility of using the threshold corrections to facilitate unification of the first and second family Yukawa couplings in the framework of supersymmetric $\mathrm{SU}(5)$. In ref. [8] trilinear soft terms proportional to the corresponding Yukawa matrices were considered. In such a case, it turned out impossible to obtain simultaneous unification for more than two families when the scalar masses were universal. On the other hand, when large off-diagonal trilinear terms were allowed, a strong tension between the unification requirement and the experimental limits on Flavour Changing Neutral Current (FCNC) processes appeared. To avoid these problems, the proportionality assumption was abandoned in ref. [9] where general diagonal $A$-terms were considered. Consequently, the threshold corrections were driven by large values of the corresponding trilinear couplings, leading to successful Yukawa unification for all three families. A similar scenario was considered in ref. [10] which updated the previous analysis in view of the Higgs boson discovery and strengthened experimental limits on the superpartner masses. With the SUSY particles getting heavier, tensions with flavour physics become weaker. It was confirmed that the SU(5) Yukawa coupling unification of all three families is phenomenologically viable and attainable for a wide range of $\tan \beta$. However, it comes at the price of a long-lived but metastable MSSM vacuum.

In the present paper, we explore an alternative scenario. We assume that the diagonal entries of the trilinear terms have the same hierarchy as the Yukawa couplings. However, we allow for non-zero off-diagonal entries both in the trilinear terms and in the sfermion mass matrices. We employ the chirally enhanced MSSM threshold corrections to fermion masses as collected in ref. [11] and previously calculated in refs. [12-14]. The most important feature of this type of corrections is that they allow to "transmit" a large $Y_{b}$-driven threshold correction to the bottom quark mass to the strange quark mass as well, provided offdiagonal flavour-violating entries in the soft mass matrices are present. We show that in order to achieve a satisfactory Yukawa coupling unification for all three families, all of the off-diagonal elements in the down-squark mass matrix are required to be non-zero, as well as the $(1,2)$ and $(2,1)$ entries in the down-sector trilinear term. This way we provide evidence that a more general treatment of the flavour structure of the renormalizable $R$-parity conserving MSSM can contribute to fulfilling the SU(5) GUT-scale boundary conditions.

The phenomenology of models with SU(5) symmetry at the GUT scale and General Flavour Violation (GFV) in the squark mass matrices has been studied in various contexts. Ref. [15-17] analysed possible signatures of their spectra at the LHC. Ref. [18] investigated properties of the dark matter (DM) candidate, while the consequences for the Higgs mass, $B$-physics and electroweak (EW) observables were discussed in refs. [19-23].

In the present study, we perform a full phenomenological analysis of the GFV SU(5) model in which the Yukawa matrix unification constraint is satisfied, taking into account such experimental measurements as the mass and the rates of the lightest Higgs boson, EW precision tests, flavour observables, relic density of the neutralino dark matter, spinindependent proton-neutralino scattering cross-section, as well as the $8 \mathrm{TeV}$ LHC exclusion bounds from the direct SUSY searches. We find that the SU(5) Yukawa unification condition is consistent at $3 \sigma$ with all the considered experimental constraints. On the other hand, a tension arises with the stability condition of the EW vacuum that turns out to be 
metastable, though long-lived. However, it should be emphasised that the tension is much weaker than in the case of large diagonal $A$-terms [10].

The phenomenological features of the scenario discussed in our study make it an attractive alternative to models assuming Minimal Flavour Violation. First of all, the FCNC processes triggered by the chirality-preserving mixing between the second and the third generation of down-type squarks, which plays a crucial role in the successful Yukawa coupling unification, are less constrained than those driven by other off-diagonal entries. Additionally, in the considered scenario, SUSY contributions to flavour observables remain relatively small thanks to low values of $\tan \beta$ and heaviness of the squarks. We also show that the lightest neutralino must be almost purely bino-like, and have mass in the range of $150-300 \mathrm{GeV}$. In the framework of the minimal $\mathrm{SU}(5)$ with gaugino unification, the lightest chargino will thus be around $350-550 \mathrm{GeV}$. Since the proper value of the DM relic density is in this case obtained through co-annihilation with the electron sneutrino, at least one generation of sleptons must be light. Interestingly, SUSY spectra of this kind have started to be probed by the LHC at $\sqrt{s}=8 \mathrm{TeV}$ in searches for events involving three leptons in the final state, and they will be completely tested at $\sqrt{s}=14 \mathrm{TeV}$.

The article is organised as follows. In section 2, we discuss SUSY threshold corrections to the Yukawa couplings in the presence of flavour-violating soft squark mass matrices. In section 3, the impact of off-diagonal soft entries on the Yukawa unification is analysed numerically, and the parameter space favoured by successful unification is determined. In section 4, we study thoroughly the phenomenology of our Yukawa unification scenario in light of the available experimental data. We summarize our findings in section 5 .

\section{Anatomy of the minimal SU(5) Yukawa unification}

Our analysis is performed in a setting that can shortly be summarized in terms of renormalization scales. The low-energy Yukawa couplings are fixed in the Standard Model, below a scale where it is matched with the MSSM (henceforth named $\mu_{s p}$ ), and at which the supersymmetric threshold corrections are calculated. Validity of the MSSM extends up to $M_{\mathrm{GUT}}$, where the minimal SU(5) boundary conditions are imposed on its parameters.

The simplest realization of the grand unification idea is based on $\mathrm{SU}(5)$, as this is the smallest symmetry to encompass the SM gauge group. In its supersymmetric version, the MSSM superfields $Q, U, D, L, E$ are embedded into the 5- and 10-dimensional representations of $\mathrm{SU}(5)$ as

$$
\begin{array}{r}
\underbrace{\left(\overline{\mathbf{3}}, \mathbf{1}, \frac{1}{3}\right)}_{D} \oplus \underbrace{\left(\mathbf{1}, \mathbf{2},-\frac{1}{2}\right)}_{L}=\underbrace{\overline{\mathbf{5}}}_{\Psi_{\overline{5}}} \\
\underbrace{\left(\mathbf{3}, \mathbf{2}, \frac{1}{6}\right)}_{Q} \oplus \underbrace{\left(\overline{\mathbf{3}}, \mathbf{1},-\frac{2}{3}\right)}_{U} \oplus \underbrace{(\mathbf{1}, \mathbf{1}, 1)}_{E}=\underbrace{\mathbf{1 0}}_{\Psi_{10}},
\end{array}
$$


where we use the conventional SM normalization for the hypercharges. The Yukawa terms for SU(5) GUT read [1]

$$
\mathcal{W} \ni \Psi_{10} \mathbf{Y}^{d e} \Psi_{\overline{5}} H_{\overline{5}}+\Psi_{10} \mathbf{Y}^{u} \Psi_{10} H_{5}
$$

where $H_{\overline{5}}$ and $H_{5}$ denote two Higgs multiplets that are coupled to matter. From eq. (2.3) it follows that all parameters that allow to determine the masses of the SM fermions are encoded in two independent $3 \times 3$ matrices $\mathbf{Y}^{d e}$ and $\mathbf{Y}^{u}$. Below the GUT scale $M_{\mathrm{GUT}} \simeq$ $2 \times 10^{16} \mathrm{GeV}$, the $\mathrm{SU}(5)$ model is replaced with the MSSM, and the effective superpotential is given by

$$
\mathcal{W}_{\mathrm{MSSM}}=Q \mathbf{Y}^{u} U H_{u}+Q \mathbf{Y}^{d} D H_{d}+L \mathbf{Y}^{e} E H_{d}+\mu H_{d} H_{u}
$$

A straightforward consequence of the GUT symmetry is the equality of the matrices $\mathbf{Y}^{d}$ and $\mathbf{Y}^{e T}$ at $M_{\mathrm{GUT}}$. This condition is true up to a basis redefinition and possible one-loop threshold corrections at this scale. In the present study, we are mainly interested in lowenergy properties of successful unification scenarios rather than in the exact realization of their high-energy UV completion. For this reason, in the subsequent analysis, we are going to allow for moderate threshold corrections at the GUT scale (see eq. (3.1)) without investigating their origin.

The unification conditions for the Yukawa couplings of down-type quarks and charged leptons take the simplest form in a basis where the superpotential flavour mixing is entirely included in $\mathbf{Y}^{u}$, while $\mathbf{Y}^{d}$ and $\mathbf{Y}^{e}$ are real and diagonal. In such a case, it is enough to require the equality of the diagonal entries at the GUT scale,

$$
Y_{i i}^{d}=Y_{i i}^{e}, \quad i=1,2,3 .
$$

\subsection{Flavour-violating threshold corrections to the Yukawa matrices at the decoupling scale}

Diagonal entries of the Yukawa couplings are constrained by measurements of the quark and lepton masses that are performed at or below the electroweak scale. Consequently, these entries are most easily fixed within the SM. One needs, however, to determine their renormalized values within the MSSM, which is assumed to be an underlying effective theory that connects the electroweak scale with $M_{\mathrm{GUT}}$. This is done by calculating threshold corrections $\Sigma_{i i}^{f}$ at the matching scale $\mu_{s p}$. Such corrections depend on values of the soft SUSY-breaking terms,

$$
v_{f} Y_{i i}^{f \mathrm{MSSM}}=v_{f} Y_{i i}^{f \mathrm{SM}}-\Sigma_{i i}^{f}\left(\left(m_{\tilde{f}}^{2}\right)_{i j}, A_{i j}^{f}, m_{H_{i}}, M_{i}\right) .
$$

The Yukawa coupling values at $M_{\mathrm{GUT}}$ are then determined by solving their MSSM renormalization group equations (RGEs) which do not depend on the soft parameters. Once this is done, the quality of the Yukawa unification for a given set of parameters can be tested.

The experimentally measured values of fermion masses can give a qualitative feeling about the problems encountered in achieving the full Yukawa matrix unification. As is well known, the constraint $Y_{b}\left(M_{\mathrm{GUT}}\right)=Y_{\tau}\left(M_{\mathrm{GUT}}\right)$ can be satisfied without large threshold 
corrections at $\mu_{\mathrm{sp}}$, at least for moderate $\tan \beta$. On the other hand, achieving strict unification of the Yukawa couplings for the remaining families $\left(Y_{s}\left(M_{\mathrm{GUT}}\right)=Y_{\mu}\left(M_{\mathrm{GUT}}\right)\right.$ and $\left.Y_{d}\left(M_{\mathrm{GUT}}\right)=Y_{e}\left(M_{\mathrm{GUT}}\right)\right)$ requires the threshold corrections to be of the same order as the leading terms. To satisfy the minimal SU(5) boundary conditions on Yukawas, the MSSM strange quark mass has to be raised w.r.t. the SM one, whereas the down-quark has to be made lighter by the threshold corrections. That does not contradict perturbativity of the model, as the corresponding leading terms are small enough, $Y_{s, d}^{(0)} \ll 1$.

The dominant supersymmetric threshold corrections to the Yukawa couplings beyond the small $\tan \beta$ limit have been calculated in ref. [11]. It was shown that in the SUSYdecoupling limit, the chirality-flipping parts of the renormalized quark (lepton) self energies $\Sigma$ are linear functions of the Yukawa couplings, with a proportionality factor $\epsilon$ and an additive term $\Sigma_{Y}$,

$$
m_{i}^{d(\ell) \mathrm{SM}}-v_{d} Y_{i i}^{d(\ell) \mathrm{MSSM}}=\Sigma_{i i}^{d(\ell) \mathrm{LR}}=\Sigma_{i i X}^{d(\ell) \mathrm{LR}}+\epsilon_{i}^{d(\ell)} v_{u} Y_{i i}^{d(\ell)(0)}+O\left(\frac{v^{2}}{M_{\mathrm{SUSY}}}\right)
$$

where $M_{\mathrm{SUSY}}$ is defined as $M_{\mathrm{SUSY}}=\sqrt{m_{\tilde{t}_{1}} m_{\tilde{t}_{2}}}$. In this approximation, the relation can easily be inverted, and the corrected MSSM Yukawa couplings in the super-CKM basis read

$$
Y_{i i}^{d(\ell) \mathrm{MSSM}}=\frac{m_{i}^{d(\ell) \mathrm{SM}}-\Sigma_{i i X^{d(\ell) \mathrm{LR}}}^{d(\ell)} .}{v_{d}\left(1+\tan \beta \cdot \epsilon_{i}\right)} .
$$

Various contributions to $\Sigma_{i i}^{d(\ell) \mathrm{LR}}$ from sfermion-gluino, sfermion-neutralino and sfermionchargino diagrams, as well as the threshold corrections to the CKM matrix can be found in ref. [11]. Their non-trivial dependence on the GFV parameters is encoded in the term $\Sigma_{Y}$. The off-diagonal soft mass elements enter eq. (2.7) through rotation matrices that diagonalise the sfermion mass matrices. On the other hand, the off-diagonal trilinears appear explicitly but give no contribution when the rotation matrices are diagonal.

To get some intuition about the functional dependence of eq. (2.7) on various entries in the sfermion mass matrices, let us consider, for example, the threshold corrections to the self-energy of down-type quarks. The most significant contribution comes from a gluino loop, as it is enhanced by the large value of the strong gauge coupling. If all the flavourviolating mass matrix entries were zero (the relevant diagram in the strange quark case is depicted in the left panel of figure 1), threshold corrections to the Yukawa couplings from a gluino loop would be given by the corresponding diagonal entries of the trilinear terms (see ref. [11]),

$$
\left(\Sigma_{i i}^{d}\right)^{\tilde{g}}=\frac{2 \alpha_{S}}{3 \pi} m_{\tilde{g}}\left(v_{d} A_{i i}^{d}-v_{d} Y_{i i}^{d} \mu \tan \beta\right) C_{0}\left(m_{\tilde{g}}^{2}, m_{\tilde{q}_{i}^{L}}^{2}, m_{\tilde{d}_{i}^{R}}^{2}\right),
$$

where the loop function $C_{0}\left(m_{\tilde{g}}^{2}, m_{\tilde{q}_{i}^{L}}^{2}, m_{\tilde{d}_{i}^{R}}^{2}\right)$ of dimension mass ${ }^{-2}$ can be found, e.g., in the appendix of ref. [11]. Interestingly, for the third family the expression given by eq. (2.9) tends to cancel with a contribution mediated by the higgsino exchange, which makes the ratio $Y_{b} / Y_{\tau}$ quite stable with respect to the SUSY threshold corrections. On the contrary, for the first and second generation the gluino contribution is dominant and can be used 


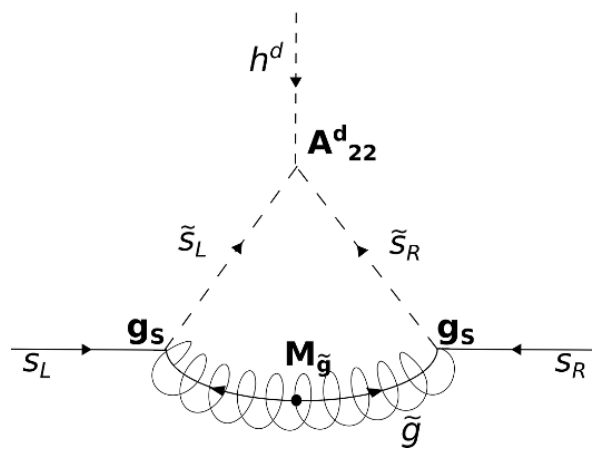

(a)

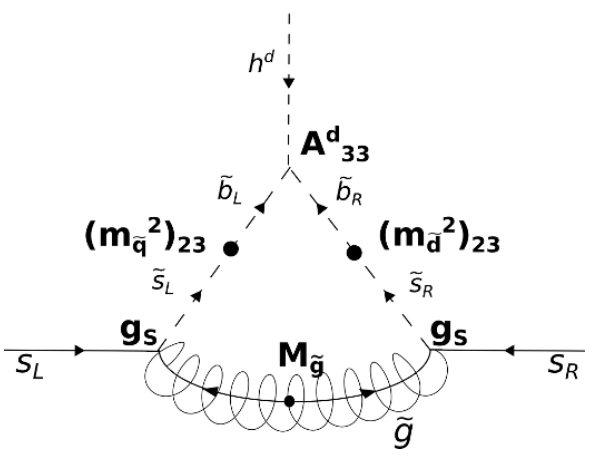

(b)

Figure 1. Examples of diagrams that describe threshold corrections to the strange Yukawa coupling at $\mu_{\mathrm{sp}}$. (a) the flavour-diagonal leading gluino diagram; (b) possibly an even bigger contribution arising in the case of flavour mixing in the soft-mass matrix.

to fix the ratios of the corresponding Yukawa couplings at $\mu_{\mathrm{sp}}$. Such a possibility was considered in refs. [8-10].

Since $C_{0}$ is always negative, it results immediately from eq. (2.9) that $A_{22}^{d}$ should be positive and $\mu$ negative to maximize the necessary positive correction to $m_{s}^{\mathrm{SM}}$ in the numerator on the r.h.s. of eq. (2.8). In the first family case, a contribution from the $\mu$ term is negligible due to smallness of the corresponding Yukawa coupling. Therefore, a negative $A_{11}^{d}$ is sufficient to generate a correction to $Y_{d}$ of the right sign.

The situation changes when non-zero off-diagonal soft mass entries are allowed (see the right panel of figure 1). In such a case, the dominant GFV contribution to the strange quark self-energy associated with a gluino loop can be written as [11]:

$$
\begin{aligned}
\left(\Sigma_{22}^{d}\right)^{\tilde{g}}=\frac{2 \alpha_{S}}{3 \pi} & m_{\tilde{g}} v_{d}\left(A_{33}^{d}-Y_{b} \mu \tan \beta\right) \sum_{m, n=2,3} C_{0}\left(m_{\tilde{g}}^{2}, m_{\tilde{q}_{m}^{L}}^{2}, m_{\tilde{d}_{n}^{R}}^{2}\right) \times \\
& \frac{\left(m_{\tilde{q}}^{2}\right)_{23}}{\sqrt{\left[\left(m_{\tilde{q}}^{2}\right)_{22}-\left(m_{\tilde{q}}^{2}\right)_{33}\right]^{2}+4\left(\left(m_{\tilde{q}}^{2}\right)_{23}\right)^{2}}} \frac{\left(m_{\tilde{d}}^{2}\right)_{23}}{\sqrt{\left[\left(m_{\tilde{d}}^{2}\right)_{22}-\left(m_{\tilde{d}}^{2}\right)_{33}\right]^{2}+4\left(\left(m_{\tilde{d}}^{2}\right)_{23}\right)^{2}}},
\end{aligned}
$$

where we have assumed that $\left(m_{\tilde{q}}^{2}\right)_{23}$ and $\left(m_{\tilde{d}}^{2}\right)_{23}$ are the only non-zero off-diagonal elements of the down-squark mass matrix, and that they are real. It follows from eq. (2.10) that chirality-conserving GFV interactions $\left(m_{\tilde{q}}^{2}\right)_{23} \tilde{b}_{L}^{*} \tilde{s}_{L}$ and $\left(m_{\tilde{d}}^{2}\right)_{23} \tilde{b}_{R}^{*} \tilde{s}_{R}$ generate a threshold correction to $Y_{s}$ of the order of $\Delta Y_{s} \sim \alpha_{S} A_{33}^{d} / M_{\mathrm{SUSY}}$, which in general can be large enough to facilitate the Yukawa coupling unification for the second family, even when the coupling $A_{22}^{d}$ is small.

The above description, however, should be treated only as a simplified qualitative illustration. In a general case, also other off-diagonal elements of the squark mass matrix can significantly differ from zero, which renders mixing among all the three generations important. To make sure that all such effects are properly taken into account, in the following we turn to a full numerical analysis. 


\begin{tabular}{|c|c|c|c|c|c|c|c|}
\hline \multicolumn{2}{|c|}{$m_{t}^{\text {pole }}$} & \multicolumn{2}{c|}{$m_{b}^{\overline{M S}}\left(m_{b}\right)$} & \multicolumn{2}{c|}{$\alpha_{s}^{\overline{M S}}\left(M_{Z}\right)$} & \multicolumn{2}{c|}{$\alpha_{\text {em }}^{-1}\left(M_{Z}\right)$} \\
$173.34 \pm 0.76 \mathrm{GeV}$ & \multicolumn{2}{|c|}{$4.18 \pm 0.03 \mathrm{GeV}$} & \multicolumn{2}{c|}{$0.1184 \pm 0.0007$} & \multicolumn{2}{|c|}{$127.944 \pm 0.015$} \\
\hline$m_{u}^{\overline{M S}}$ & $m_{d}^{\overline{M S}}$ & $m_{s}^{\overline{M S}}$ & $m_{c}^{\overline{M S}}\left(m_{c}\right)$ & $m_{e}^{\text {pole }}$ & $m_{\mu}^{\text {pole }}$ & $m_{\tau}^{\text {pole }}$ & $M_{Z}^{\text {pole }}$ \\
$2.3 \mathrm{MeV}$ & $4.8 \mathrm{MeV}$ & $95 \mathrm{MeV}$ & $1.275 \mathrm{GeV}$ & $511 \mathrm{keV}$ & $106 \mathrm{MeV}$ & $1.777 \mathrm{GeV}$ & $91.19 \mathrm{GeV}$ \\
\hline \multicolumn{2}{|c|}{$\bar{\rho}$} & \multicolumn{2}{|c|}{$\bar{\eta}$} & \multicolumn{2}{|c|}{$A$} & \multicolumn{2}{|c|}{$\lambda$} \\
$0.159 \pm 0.045$ & \multicolumn{2}{|c|}{$0.363 \pm 0.049$} & \multicolumn{2}{c|}{$0.802 \pm 0.020$} & \multicolumn{2}{c|}{$0.22535 \pm 0.00065$} \\
\hline
\end{tabular}

Table 1. Standard Model parameters [27, 28] used in our numerical calculations. The light $(u, d, s)$ quark masses are $\overline{\mathrm{MS}}$-renormalized at $2 \mathrm{GeV}$.

\section{Impact of the GFV threshold corrections on Yukawa unification}

Let us start this section with briefly describing the numerical tools and procedures employed to identify the GFV SU(5) GUT parameter space regions where the Yukawa coupling unification for all the three families becomes possible. Next, we shall perform a numerical scan and use its results to determine those SUSY parameters which non-zero values are indispensable from the point of view of the considered unification.

\subsection{Numerical tools and scanning methodology}

Points satisfying the Yukawa coupling unification conditions at the GUT scale are found by scanning the parameter space of the model defined in section 3.2. To this end, we use the numerical package BayesFITSv3.2 that was first developed in ref. [24] and modified to incorporate the full GFV structure of the soft SUSY-breaking sector in ref. [23]. The package uses for sampling MultiNest v2.7 [25] which allows for fast and efficient scanning according to a predefined likelihood function. The likelihood corresponding to the SU(5) boundary condition (2.5) is modeled with a Gaussian distribution as follows,

$$
\mathcal{L}_{Y u k}=\sum_{i=1,2,3} \exp \left[-\left(1-Y_{i i}^{e}\left(M_{\mathrm{GUT}}\right) / Y_{i i}^{d}\left(M_{\mathrm{GUT}}\right)\right)^{2} / 2 \sigma_{Y u k}^{2}\right]
$$

and the allowed deviation from the exact unification condition, $\sigma_{Y u k}$, is set to $5 \%$.

Mass spectra are calculated with SPheno v3.3.3 [26]. The choice is dictated by the fact that at the moment SPheno is the only SUSY spectrum generator where the full flavour structure of threshold corrections to the Yukawa couplings, as given in ref. [11], is implemented. As is the case of other tools of this kind, the renormalization group equations of the MSSM are solved by an iterative algorithm that interpolates between different scales at which the parameters are defined. The boundary with the SM (i.e. the scale $\mu_{\mathrm{sp}}$ ) is set at $M_{Z}$.

Four SM parameters $\left(m_{t}^{\text {pole }}, m_{b}^{\overline{M S}}\left(m_{b}\right), \alpha_{\mathrm{em}}^{-1}\left(M_{Z}\right)\right.$ and $\left.\alpha_{s}^{\overline{M S}}\left(M_{Z}\right)\right)$ are treated as nuisance parameters and randomly drawn from a Gaussian distribution centred around their experimentally measured values [27]. The elements of the CKM matrix in the Wolfenstein parametrisation $(\bar{\rho}, \bar{\eta}, A, \lambda)$ are scanned as well, with central values and errors given by the 
UTfit Collaboration for the scenario allowing new physics effects in loop observables [28]. The other SM parameters which are passed as an input to SPheno $\left(m_{s}^{\overline{M S}}, m_{c}^{\overline{M S}}\left(m_{c}\right), m_{d}^{\overline{M S}}\right.$, $\left.m_{u}^{\overline{M S}}, m_{\tau}^{\text {pole }}, m_{\mu}^{\text {pole }}, m_{e}^{\text {pole }}, M_{Z}^{\text {pole }}\right)$ are fixed at their experimentally measured values. Our $\mathrm{SM}$ input is collected in table 1 .

\subsection{Input SUSY parameters}

We start our analysis by defining a general set of SUSY parameters at the GUT scale. Since a priori we do not know which GFV parameters in the down-squark sector are indispensable to achieve the Yukawa coupling unification and which can be skipped, initially we allow all of them to assume non-zero values. The aim of the numerical scan is to identify those parameters that are essential.

We assume for simplicity that all the soft SUSY-breaking parameters are real, therefore neglecting the possibility of new SUSY sources of CP violation. The GUT-scale SU(5) boundary conditions for the soft-masses read ${ }^{1}$

$$
\left(m_{\tilde{l}}^{2}\right)_{i j}=\left(m_{\tilde{d}}^{2}\right)_{i j} \equiv\left(m_{d l}^{2}\right)_{i j}, \quad\left(m_{\tilde{q}}^{2}\right)_{i j}=\left(m_{\tilde{u}}^{2}\right)_{i j}=\left(m_{\tilde{e}}^{2}\right)_{i j} \equiv\left(m_{u e}^{2}\right)_{i j} .
$$

We do not impose any additional conditions on the relative magnitudes of the diagonal entries. In particular, both normal and inverted hierarchies for the elements $\left(m_{d l}^{2}\right)_{i i}$ and $\left(m_{u e}^{2}\right)_{i i}$ are allowed. The off-diagonal elements of the down-squark matrix are normalized to the entry $\left(m_{d l}^{2}\right)_{33}$, and are required to satisfy the upper limit $\left(m_{d l}^{2}\right)_{i j} /\left(m_{d l}^{2}\right)_{33} \leq 1$.

We further assume that

$$
\left(m_{u e}^{2}\right)_{i j}=0, \quad i \neq j .
$$

Such an assumption is not expected to cause any significant loss of generality because relatively large off-diagonal elements of $\left(m_{\tilde{q}}^{2}\right)_{i j}$ are generated radiatively at $M_{\text {SUSY }}$ due to the RGE running in the super-CKM basis. Note also that $\left(m_{q}^{2}\right)_{23}\left(M_{\mathrm{SUSY}}\right)>0$ and $\left(m_{q}^{2}\right)_{13}\left(M_{\mathrm{SUSY}}\right)<0$ are desired for the Yukawa unification. When such inequalities are satisfied, the correction in eq. (2.10) turns out to be negative for the strange quark and positive for the down quark, as long as the trilinear term $A_{33}^{d l}$ is greater than zero. For the same reason we also restrict our study to the case $\left(m_{d l}^{2}\right)_{i j}>0$. At this point we are allowed to introduce a short-hand notation

$$
m_{i j}^{d l} \equiv \sqrt{\left(m_{d l}^{2}\right)_{i j}}, \quad m_{i j}^{u e} \equiv \sqrt{\left(m_{u e}^{2}\right)_{i j}} .
$$

The GUT-scale SU(5) boundary conditions for the trilinear terms are given by

$$
A_{i j}^{d}=A_{i j}^{e} \equiv A_{i j}^{d e}
$$

We constrain the relative magnitude of the diagonal entries by the corresponding Yukawa couplings

$$
\frac{\left|A_{i i}^{f}\right|}{\left|A_{33}^{f}\right|}<\frac{Y_{i i}^{f}}{Y_{33}^{f}}
$$

\footnotetext{
${ }^{1}$ These relations have an important impact one the flavour phenomenology, see "Note added in proof".
} 


\begin{tabular}{|c|c|}
\hline Parameter & Scanning Range \\
\hline$M_{1 / 2}$ & {$[100,4000] \mathrm{GeV}$} \\
$m_{H_{u}}$ & {$[100,8000] \mathrm{GeV}$} \\
$m_{H_{d}}$ & {$[100,8000] \mathrm{GeV}$} \\
$\tan \beta$ & {$[3,45]$} \\
$\operatorname{sgn} \mu$ & -1 \\
\hline$A_{33}^{d e}$ & {$[0,5000] \mathrm{GeV}$} \\
$A_{33}^{u}$ & {$[-9000,9000] \mathrm{GeV}$} \\
$A_{11}^{d e} / A_{33}^{d e}$ & {$[-0.00028,0.00028]$} \\
$A_{22}^{d e} / A_{33}^{d e}$ & {$[-0.065,0.065]$} \\
$A_{22}^{u} / A_{33}^{u}$ & {$[-0.005,0.005]$} \\
$A_{i j}^{d e} / A_{33}^{d e}, i \neq j$ & {$[-0.5,0.5]$} \\
\hline$m_{i i}^{d l}, i=1,2,3$ & {$[100,7000] \mathrm{GeV}$} \\
$m_{23}^{d l} / m_{33}^{d l}$ & {$[0,1]$} \\
$m_{13}^{d l} / m_{33}^{d l}$ & {$[0,1]$} \\
$m_{12}^{d l} / m_{33}^{d l}$ & {$[0,1]$} \\
$m_{i i}^{u e}, i=1,2,3$ & {$[100,7000] \mathrm{GeV}$} \\
\hline
\end{tabular}

Table 2. Ranges of the input SUSY parameters used in our initial scan. Several omitted soft SUSY-breaking parameters at the GUT scale (namely $A_{11}^{u}$ as well as $A_{i j}^{u}$ and $m_{i j}^{u e}$ for $i \neq j$ ) have been set to zero.

We do so because we aim at relaxing the strong tension between the EW vacuum stability condition and Yukawa unification that has been observed [10] in the case of large diagonal $A$-terms. We also impose that

$$
A_{i j}^{u}=0, \quad i \neq j .
$$

On the other hand, the off-diagonal entries in the down-sector trilinear matrix are not constrained to scale proportionally to the corresponding Yukawa matrix entries. They are only required to satisfy $\left|\left(A_{i j}^{d}\right) /\left(A_{33}^{d}\right)\right| \leq 0.5$.

Finally, we assume that the gaugino mass parameters are universal at $M_{\mathrm{GUT}}$,

$$
M_{1}=M_{2}=M_{3} \equiv M_{1 / 2},
$$

which is the simplest among relations that naturally arise in the framework of SUSY SU(5) GUTs. The sign of the parameter $\mu$ is chosen to be negative to facilitate the second family unification, as explained in section 2.

In table 2, we collect the scanning ranges for our initial set of input SUSY parameters. We allow quite wide ranges for the diagonal soft scalar masses, the universal gaugino mass parameter $M_{1 / 2}$ and the trilinear coupling $A_{33}^{u}$, as those are the parameters particularly important for satisfying the DM relic density and the Higgs boson mass constraints. We restrict the scanned values of $\tan \beta$ to remain below 45 , to avoid regions of the parameter 


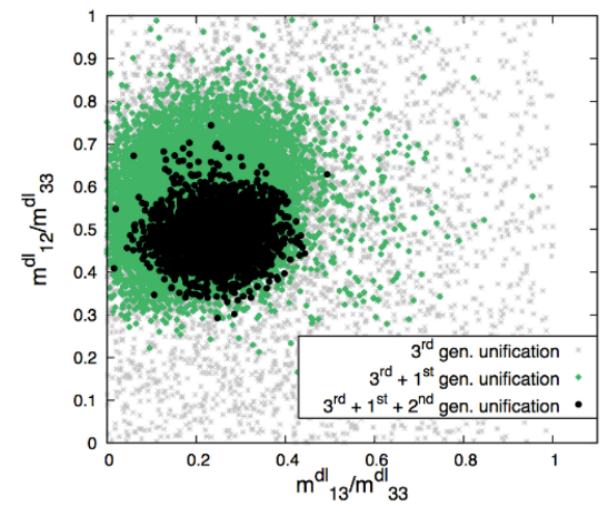

(a)

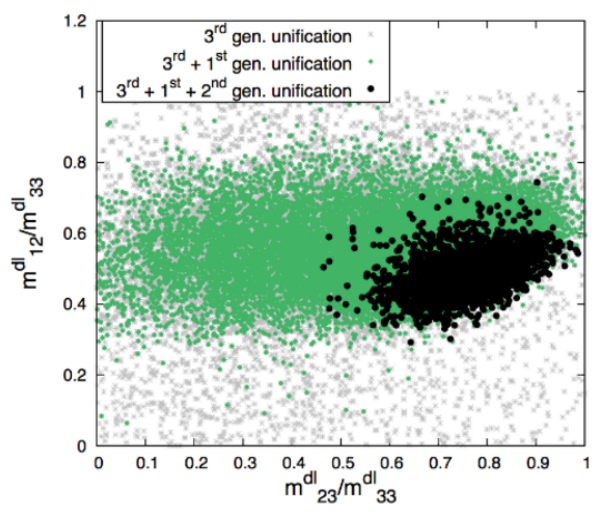

(b)

Figure 2. Scatter plot of the GFV SU(5) points in the planes $\left(m_{13}^{d l} / m_{33}^{d l}, m_{12}^{d l} / m_{33}^{d l}\right)$ (a), and $\left(m_{23}^{d l} / m_{33}^{d l}, m_{13}^{d l} / m_{33}^{d l}\right)$ (b). Gray stars: all the points satisfying the Yukawa unification condition for the third generation at $2 \sigma$; green diamonds: points additionally requiring $2 \sigma$ unification of the first family; black dots: points for which all the three generations are unified at $2 \sigma$.

space where no proper Yukawa coupling unification has occured in our preliminary scans. As far as the other input SUSY parameters are concerned, their scanning ranges have already been described above.

In the next subsection, we shall identify those SUSY parameters that are particularly relevant from the point of view of the Yukawa coupling unification.

\subsection{Yukawa unification through flavour-violating terms}

We collected about $1.5 \times 10^{5}$ points through our scanning procedure of the parameter space defined in table 2 .

In figure 2, we present distributions of points in the planes $\left(m_{13}^{d l} / m_{33}^{d l}, m_{12}^{d l} / m_{33}^{d l}\right)$ (a) and $\left(m_{23}^{d l} / m_{33}^{d l}, m_{13}^{d l} / m_{33}^{d l}\right)$ (b). All the points that satisfy the Yukawa unification condition for the third generation at $2 \sigma\left(0.9<Y_{b} / Y_{\tau}<1.1\right)$ are depicted as gray stars (they account for $49 \%$ of all the points), while those that additionally fulfil $2 \sigma$ unification of the first family as green diamonds ( $29 \%$ of all the points). Finally, black dots correspond to those points for which all three generations are unified at $2 \sigma$ (1.7\% of all the points collected by the scan). In figure 3, similar distributions are shown for the flavour-violating entries of the trilinear down-sector matrix, in the planes corresponding to $\left(A_{12}^{d e} / A_{33}^{d e}, A_{21}^{d e} / A_{33}^{d e}\right)$ (a), $\left(A_{13}^{d e} / A_{33}^{d e}, A_{31}^{d e} / A_{33}^{d e}\right)(\mathrm{b})$, and $\left(A_{23}^{d e} / A_{33}^{d e}, A_{32}^{d e} / A_{33}^{d e}\right)(\mathrm{c})$.

Several observations can now be made. First of all, it is known that a satisfactory unification of the third family Yukawa couplings is quite easy to achieve in the Minimal Flavour Violating $\mathrm{SU}(5)$ for moderate values of $\tan \beta$. This is confirmed by both figure 2 and figure 3 where gray points can easily be found for vanishing flavour-violation in the GUT-scale soft parameters.

Secondly, the functional form of the threshold correction in eq. (2.10) might suggest that non-zero soft-mass elements $m_{23}^{d l}$ and $m_{13}^{d l}$ are sufficient to allow the Yukawa unification in both the second and first family cases. Such a simplistic picture, however, is not true, 


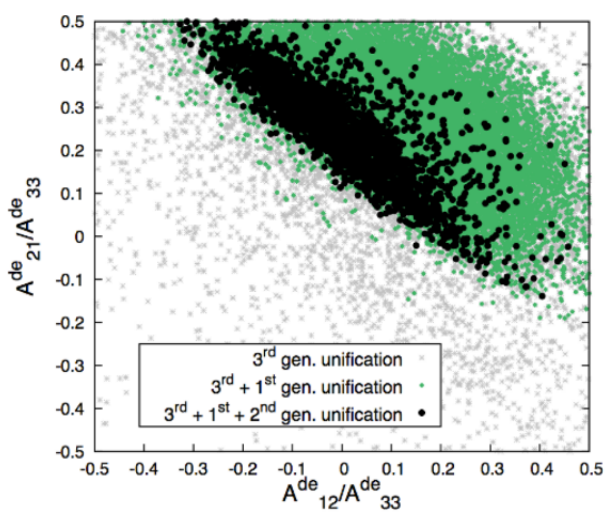

(a)

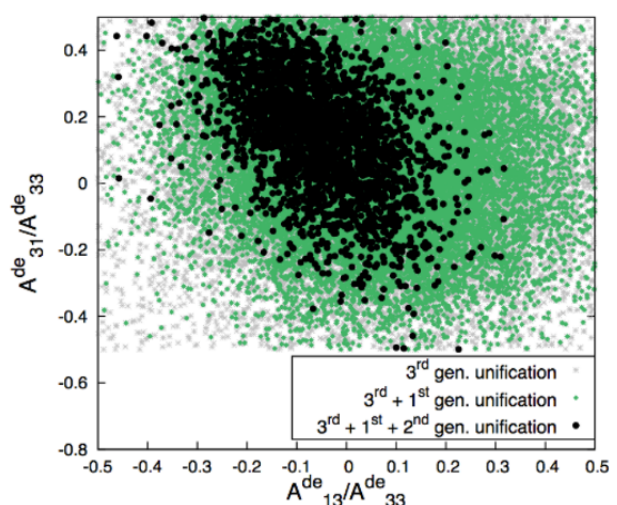

(b)

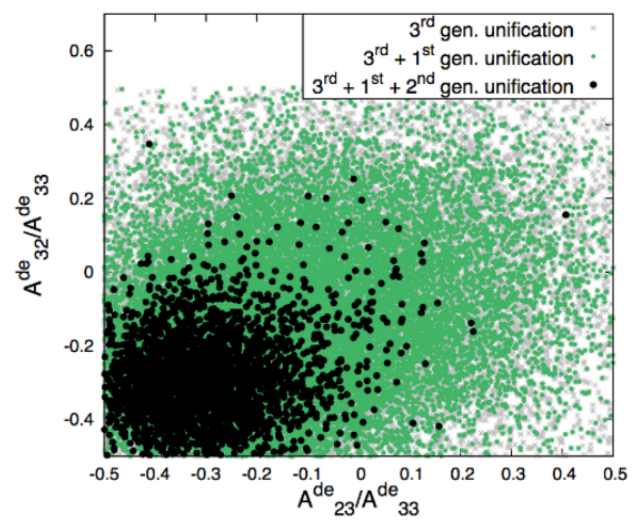

(c)

Figure 3. Scatter plot of the GFV SU(5) points in the planes $\left(A_{12}^{d e} / A_{33}^{d e}, A_{21}^{d e} / A_{33}^{d e}\right)(\mathrm{a}),\left(A_{13}^{d e} / A_{33}^{d e}\right.$, $\left.A_{31}^{d e} / A_{33}^{d e}\right)(\mathrm{b})$, and $\left(A_{23}^{d e} / A_{33}^{d e}, A_{32}^{d e} / A_{33}^{d e}\right)(\mathrm{c})$. The colour code is the same as in figure 2 .

as can be seen from the panel (a) of figure 2 where large $m_{12}^{d l}$ is clearly favoured. To understand what happens, let us note that the GFV corrections $\left(\Sigma_{22}^{d}\right)^{\tilde{g}}$ and $\left(\Sigma_{11}^{d}\right)^{\tilde{g}}$ (obtained from eq. (2.10) by replacing indices "2" with "1") are determined by overlapping sets of parameters, in particular $M_{1 / 2}$ and $A_{33}^{d e}$. On the other hand, sizes of those corrections as required by the Yukawa coupling unification differ by two orders of magnitude. Let us now assume that $\left(\Sigma_{11}^{d}\right)^{\tilde{g}}$ is fixed by the unification condition for the first family. Thus $M_{1 / 2}$ and $A_{33}^{d e}$, already constrained by unification of the third family, are even more limited. With such a choice of parameters, however, the correction $\left(\sum_{22}^{d}\right)^{\tilde{g}}$ is still too small to allow the unification of the second family, and needs to be further enhanced by another contribution. Such a contribution comes from a diagram like the one shown in figure 1(b), but with the trilinear term $A_{21}^{d e}$ in the vertex and $m_{12}^{d l}$ mixing in the right-handed sector. However, a similar diagram also exists for the first family, and the corresponding contribution should be added to the one driven by $m_{13}^{d l}$. That explains why all the five parameters $m_{12}^{d l}, m_{13}^{d l}$, $m_{23}^{d l}, A_{12}^{d e}$ and $A_{21}^{d e}$ must be adjusted simultaneously. Note also that $A_{12 / 21}^{d e}$ can be kept relatively low, as this contribution is always enhanced by a large value of $m_{12}^{d l}$. 


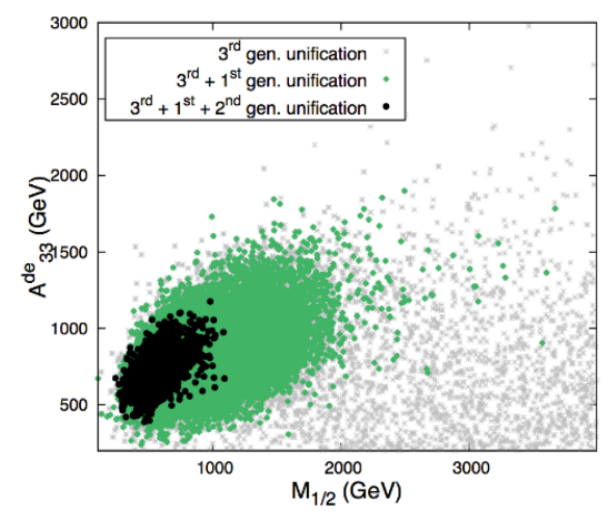

(a)

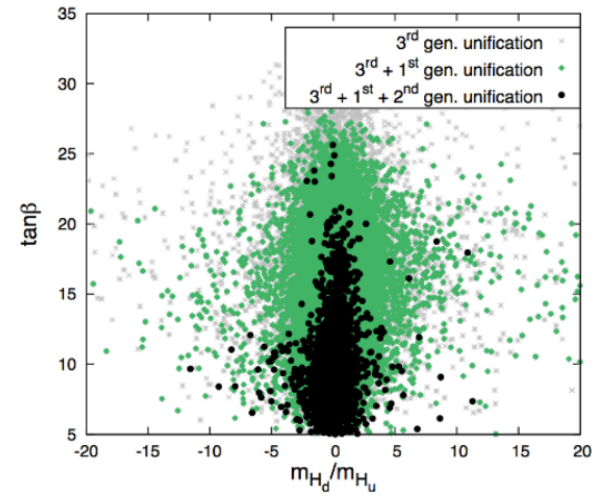

(b)

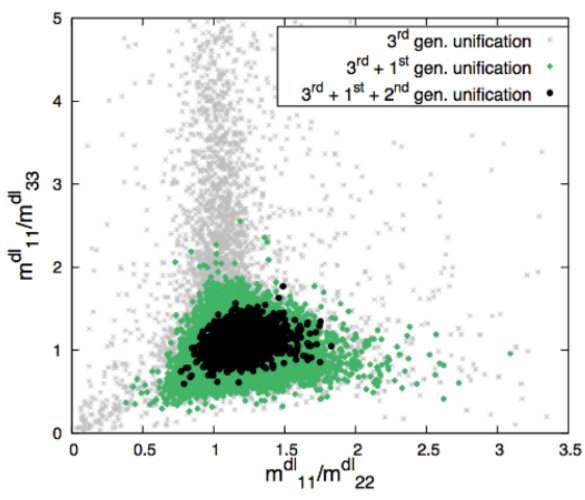

(c)

Figure 4. Scatter plot of the GFV SU(5) points in the planes $\left(M_{1 / 2}, A_{33}^{d}\right)(\mathrm{a}),\left(m_{H_{d}} / m_{H_{u}}, \tan \beta\right)$ (b), and $\left(m_{11}^{d l} / m_{22}^{d l}, m_{11}^{d l} / m_{33}^{d l}\right)(\mathrm{c})$. The colour code is the same as in figure 2 .

Flavour-violating parameters are not the only ones constrained by the Yukawa unification condition. In figure 4 , we present distributions of points in the planes $\left(M_{1 / 2}, A_{33}^{d e}\right)$ (a), $\left(m_{H_{d}} / m_{H_{u}}, \tan \beta\right)(\mathrm{b})$, and $\left(m_{11}^{d l} / m_{22}^{d l}, m_{11}^{d l} / m_{33}^{d l}\right)$ (c). The colour code is the same as in figure 2. One can observe that values of both $M_{1 / 2}$ and $A_{33}^{d}$ need to be very limited in order to facilitate unification in the first and second family cases, as they directly enter eq. (2.10). The ratio $m_{H_{d}} / m_{H_{u}}$ in the range [0-2] allows the unification of the second family for larger values of $\tan \beta, \tan \beta \in[15-25]$. Finally, large mass splittings between the diagonal entries of the down-squark mass matrix are disfavoured because they would lead to a strong suppression of SUSY threshold corrections, as can be deduced from eq. (2.10).

We conclude this section by summarising the ranges of non-zero GFV parameters that closely embed the region with full Yukawa coupling unification in the SU(5) GUT:

$$
\begin{gathered}
0.5<m_{23}^{d l} / m_{33}^{d l}<1, \quad 0<m_{13}^{d l} / m_{33}^{d l}<0.5, \quad 0.3<m_{12}^{d l} / m_{33}^{d l}<0.7, \\
0<A_{12}^{d} / A_{33}^{d}<0.2, \quad 0<A_{21}^{d} / A_{33}^{d}<0.2 .
\end{gathered}
$$

We have thus proved that it is possible to obtain $\mathrm{SU}(5)$ unification of the Yukawa matrices in the renormalizable R-parity conserving MSSM. The fact that one of the down-squark 
trilinear terms has to be non-zero is going to have significant consequences for EW vacuum stability, as discussed further in section 4.2.

\section{Phenomenology of the unification scenario}

So far, we have discussed the possibility of satisfying the $\mathrm{SU}(5)$ boundary conditions for the Yukawa couplings of all three generations by allowing a non-trivial flavour structure of the soft terms at the GUT scale. In this section, we will discuss the compatibility of the results obtained in section 3 with various phenomenological constraints. First, we are going to consider a set of experimental measurements from the dark matter searches, Higgs physics, flavour physics and EW precision measurements. Next, theoretical limits provided by the EW vacuum stability requirement will be taken into account. We will conclude this section with a list of phenomenologically realistic benchmark points that allow a satisfactory Yukawa coupling unification.

In this exposition, we do not aim at finding the best possible fit of the MSSM to the experimental data. Rather, we show that the Yukawa unification requirement does not contradict consistency of the MSSM with observations.

\subsection{Experimental constraints}

In order to find points satisfying both the Yukawa coupling unification conditions at the GUT scale and the experimental constraints, we use the tools described in section 3.1, and scan the parameter space of the unification scenario given in table 3 . The scanning ranges are chosen to cover the region consistent with a full $\mathrm{SU}(5)$ unification of the Yukawa matrices, as discussed in section 3.3. Thus, we restrict ourselves to $M_{1 / 2}$ lower than $1 \mathrm{TeV}$, $\tan \beta<25$ and $A_{33}^{d e}$ in the range $[400,1100] \mathrm{GeV}$. The off-diagonal terms $A_{12}^{d e} / A_{33}^{d e}, A_{21}^{d e} / A_{33}^{d e}$, $m_{23}^{d l} / m_{33}^{d l}, m_{13}^{d l} / m_{33}^{d l}, m_{12}^{d l} / m_{33}^{d l}$ are restricted according to eq. (3.9). For the remaining parameters, we adopt the initial scanning ranges (cf. table 2), as they do not affect the Yukawa unification. The experimental constraints applied in the analysis are listed in table 4 .

All the flavour observables have been evaluated with the code SUSY_FLAVOR v2.10 [29]. It calculates the renormalized Yukawa matrices in the MSSM and derives the correct CKM matrix as described in ref. [11]. Apart from the $B$-meson mass differences $\Delta M_{B_{d}}$ and $\Delta M_{B_{s}}$, we also consider their ratio $\Delta M_{B_{d}} / \Delta M_{B_{s}}$ for which more precise lattice inputs are available [30,31]. Theory uncertainties in the cases of $\mathrm{BR}\left(\overline{\mathrm{B}} \rightarrow \mathrm{X}_{\mathrm{s}} \gamma\right), \operatorname{BR}\left(B_{s(d)} \rightarrow \mu^{+} \mu^{-}\right)$ and $\left|\epsilon_{K}\right|$ have been estimated following refs. [32, 33], and [34], respectively. In the latter case, they arise mainly from poor convergence of the QCD perturbation series for double charm contributions. Errors stemming from the Wolfenstein parameters $\rho$ and $\eta$ are not taken into account in the third column of table 4. Each point of our scan corresponds to a particular value of $(\rho, \eta)$, and it is tested against SUSY-sensitive loop observables that matter for determining allowed regions in the $(\rho, \eta)$ plane. At the same time (as we have already mentioned in section 3.1), our initial ranges for $\rho$ and $\eta$ are adopted from the so-called 'new physics fit' by the UTFit collaboration [28]. 


\begin{tabular}{|c|c|}
\hline Parameter & Scanning Range \\
\hline$M_{1 / 2}$ & {$[200,1100] \mathrm{GeV}$} \\
$m_{H_{u}}, m_{H_{d}}$ & {$[100,8000] \mathrm{GeV}$} \\
$\tan \beta$ & {$[3,25]$} \\
$\operatorname{sgn} \mu$ & -1 \\
\hline$A_{33}^{d e}$ & {$[400,1100] \mathrm{GeV}$} \\
$A_{33}^{u}$ & {$[-9000,9000] \mathrm{GeV}$} \\
$A_{11}^{d e} / A_{33}^{d e}$ & {$[-0.00028,0.00028]$} \\
$A_{22}^{d e} / A_{33}^{d e}$ & {$[-0.065,0.065]$} \\
$A_{22}^{u} / A_{33}^{u}$ & {$[-0.005,0.005]$} \\
$A_{12}^{d e} / A_{33}^{d e}, A_{21}^{d e} / A_{33}^{d e}$ & {$[-0.2,0.2]$} \\
\hline$m_{i i}^{d l}, i=1,2,3$ & {$[100,7000] \mathrm{GeV}$} \\
$m_{23}^{d l} / m_{33}^{d l}$ & {$[0.5,1.0]$} \\
$m_{13}^{d l} / m_{33}^{d l}$ & {$[0.0,0.5]$} \\
$m_{12}^{d l} / m_{33}^{d l}$ & {$[0.3,0.7]$} \\
$m_{i i}^{u e}, i=1,2,3$ & {$[100,7000] \mathrm{GeV}$} \\
\hline
\end{tabular}

Table 3. Ranges of the input SUSY parameters in the GFV SU(5) scenario with Yukawa coupling unification. Several parameters that are not explicitly listed in the table (namely $A_{11}^{u}, A_{i j}^{u}$ and $m_{i j}^{u e}$ for $i \neq j, A_{23 / 32}^{d e}, A_{13 / 31}^{d e}$ ) have been set to zero.

The relic density and the spin-independent neutralino-proton cross section $\sigma_{p}^{\mathrm{SI}}$ have been calculated with the help of DarkSUSY v5.0.6 [35]. For the EW precision constraints FeynHiggs v2.10.0 [36-39] has been used. To include the exclusion limits from Higgs boson searches at LEP, Tevatron, and LHC, as well as the $\chi^{2}$ contributions from the Higgs boson signal rates from Tevatron and LHC, we applied HiggsBounds v4.0.0 [40-42] interfaced with HiggsSignals v1.0.0 [43].

In all the cases, the likelihood for positive experimental measurements was modeled with Gaussian distribution, while for upper bounds - with half-Gaussian one. The likelihood relative to the LUX results [44] was calculated by closely following the procedure first developed in ref. [45] and described in section 3 of ref. [46]. The likelihood for the LHC direct SUSY searches was not included in the global likelihood used by MultiNest. Our treatment of this constraint will be described in section 4.1.3. We collected in total about $2.1 \times 10^{5}$ points.

\subsubsection{Dark matter}

The relic abundance of the dark matter in GUT-constrained SUSY scenarios is usually the most stringent constraint on their parameter space. It is well known that the properties of the DM candidate strongly depend on its composition. When the lightest supersymmetric particle (LSP) is almost purely bino, the relic density is generally too large. Therefore, 


\begin{tabular}{|l|l|l|l|}
\hline Measurement & Mean or range & Error [ exp., th.] & Reference \\
\hline$\Omega_{\chi} h^{2}$ & 0.1199 & {$[0.0027,10 \%]$} & {$[47]$} \\
LUX $(2013)$ & See section 3 of $[46]$ & See section 3 of $[46]$ & {$[44]$} \\
$m_{h}($ by CMS $)$ & $125.7 \mathrm{GeV}$ & {$[0.4,3.0] \mathrm{GeV}$} & {$[48]$} \\
$\sin ^{2} \theta_{\text {eff }}$ & 0.23155 & {$[0.00012,0.00015]$} & {$[27]$} \\
$M_{W}$ & $80.385 \mathrm{GeV}$ & {$[0.015,0.015] \mathrm{GeV}$} & {$[27]$} \\
$\mathrm{BR}\left(\overline{\mathrm{B}} \rightarrow \mathrm{X}_{\mathrm{s}} \gamma\right) \times 10^{4}$ & 3.43 & {$[0.22,0.23]$} & {$[31]$} \\
$\mathrm{BR}\left(\mathrm{B}_{\mathrm{s}} \rightarrow \mu^{+} \mu^{-}\right) \times 10^{9}$ & 2.8 & {$[0.7,0.23]$} & {$[49]$} \\
$\mathrm{BR}\left(\mathrm{B}_{\mathrm{d}} \rightarrow \mu^{+} \mu^{-}\right) \times 10^{10}$ & 3.9 & {$[1.6,0.2]$} & {$[49]$} \\
$\Delta M_{B_{s}} \times 10^{11}$ & $1.1691 \mathrm{GeV}$ & {$[0.0014,0.1580] \mathrm{GeV}$} & {$[27]$} \\
$\Delta M_{B_{d}} \times 10^{13}$ & $3.357 \mathrm{GeV}$ & {$[0.033,0.340] \mathrm{GeV}$} & {$[27]$} \\
$\Delta M_{B_{d}} / \Delta M_{B_{s}} \times 10^{2}$ & 2.87 & {$[0.02,0.14]$} & {$[31]$} \\
$\sin (2 \beta)_{\exp }$ & 0.682 & {$[0.019,0.003]$} & {$[27]$} \\
$\mathrm{BR}\left(\mathrm{B}_{\mathrm{u}} \rightarrow \tau \nu\right) \times 10^{4}$ & 1.14 & {$[0.27,0.07]$} & {$[27]$} \\
$\mathrm{BR}\left(K^{+} \rightarrow \pi^{+} \nu \bar{\nu}\right) \times 10^{10}$ & 1.73 & {$[1.15,0.04]$} & {$[27]$} \\
$\left|d_{n}\right| \times 10^{26}$ & $<2.9 e \mathrm{~cm}$ & {$[0,30 \%]$} & {$[50]$} \\
$\epsilon_{K} \times 10^{3}$ & 2.228 & {$[0.011,0.17]$} & {$[27]$} \\
$\mathrm{LHC} \sqrt{s}=8 \mathrm{TeV}$ & section 4.1 .3 & section 4.1 .3 & {$[51-53]$} \\
\hline
\end{tabular}

Table 4. The experimental constraints applied in the analysis.

the annihilation cross-section needs to be enhanced by some mechanism, usually by coannihilation with the lightest sfermion, or resonance annihilation through one of the Higgs bosons. On the other hand, a significant higgsino component of the lightest neutralino opens the possibility of efficient annihilation into gauge bosons through a $t$-channel exchange of higgsino-like $\tilde{\chi}_{1}^{ \pm}$and $\tilde{\chi}_{2}^{0}$. In fact, the annihilation cross-section in such a case is usually too large, which leads to DM underabundance.

In figure 5 , we present distributions of points found by our scanning procedure in the planes $\left(m_{A}, \mu\right)(\mathrm{a}),\left(m_{\tilde{\chi}_{1}^{0}}, \sigma_{p}^{\mathrm{SI}}\right)(\mathrm{b})$ and $\left(\tan \beta, M_{1 / 2}\right)(\mathrm{c})$. All the points collected by the scan are depicted as gray stars, while those that satisfy at $3 \sigma$ the experimental constraint on the DM relic density appear as brown dots. Blue diamonds correspond to those scenarios for which additionally the Yukawa coupling unification holds. The green dashed line indicates the $90 \%$ C.L. exclusion bound on the $\sigma_{p}^{\text {SI }}$ based on the 85 -day measurement by the LUX collaboration [44], while the purple dashed line is a projection of XENON1T sensitivity [54]. By comparing panels (a) and (b) of figure 5 one can see that in the region where Yukawa unification is achieved, the neutralino LSP is bino-like, which corresponds to a relatively low spin-independent proton-neutralino cross-section. In other words, the condition of Yukawa coupling unification strongly disfavours purely or partly higgsino-like neutralino. This is due to the fact that the $\mu$ parameter value associated with this region is relatively 


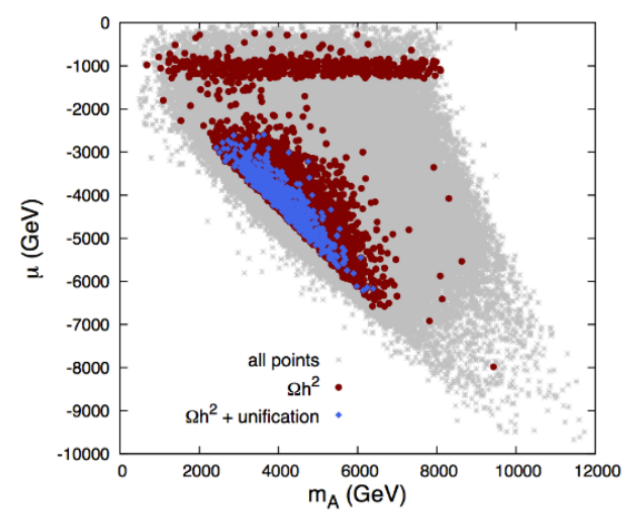

(a)

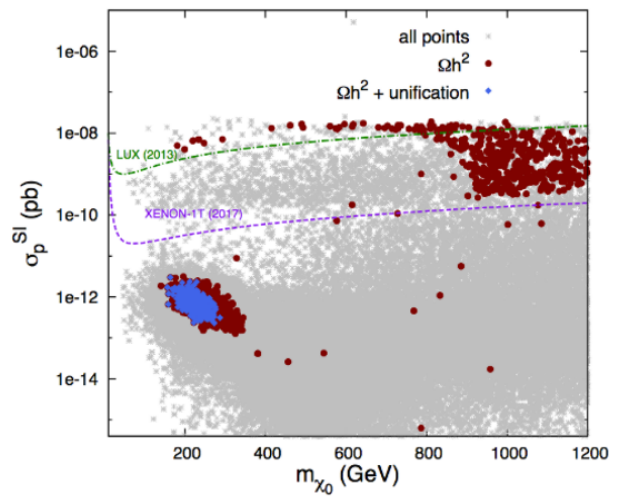

(b)

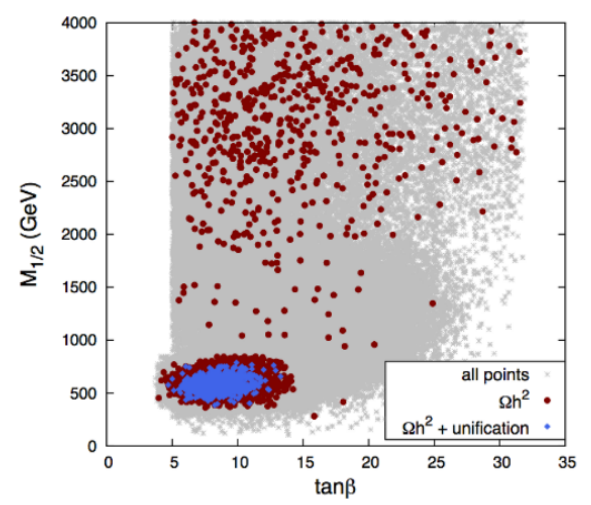

(c)

Figure 5. Scatter plot of the GFV SU(5) points in the planes $\left(m_{A}, \mu\right)(\mathrm{a}),\left(m_{\tilde{\chi}_{1}^{0}}, \sigma_{p}^{\mathrm{SI}}\right)(\mathrm{b})$ and $\left(\tan \beta, M_{1 / 2}\right)(\mathrm{c})$. Gray stars: all points collected by the scan; brown dots: points satisfying the DM relic density constraint at $3 \sigma$; blue diamonds: points for which additionally the Yukawa unification holds. The meaning of dashed lines is described in the text.

low $(\mu \lesssim 1 \mathrm{TeV})$ and the $\mu$-dependent contribution in eq. (2.10) is too small to allow the unification of the second family. Thus, it is an important phenomenological signature of the GFV SU(5) Yukawa unification scenario with the universal gaugino masses that only bino-like neutralino is allowed.

A unique mechanism that makes the effective increase of the DM annihilation crosssection possible in this case is the neutralino co-annihilation with the lightest sneutrino. The pseudoscalar is too heavy to allow a resonant $\tilde{\chi}_{1}^{0}$ annihilation (as can be read from the panel (a) of figure 5), while the masses of the coloured sfermions in the GUT-constrained unification scenarios are always larger than those of the sleptons. It is due to a renormalization effect, as their RGE running is strongly driven by the gluino. Such a property of the spectrum, however, has important consequences for experimental testability. In figure 5(b) the dashed lines indicate the present reach and the expected sensitivity for LUX and XENON1T. The region favoured by the relic density constraint in the Yukawa unification scenario remains far beyond the reach for any of them. On the other hand, spectra characterized by the presence of a light bino-like $\tilde{\chi}_{1}^{0}$ and a sneutrino close in mass are 


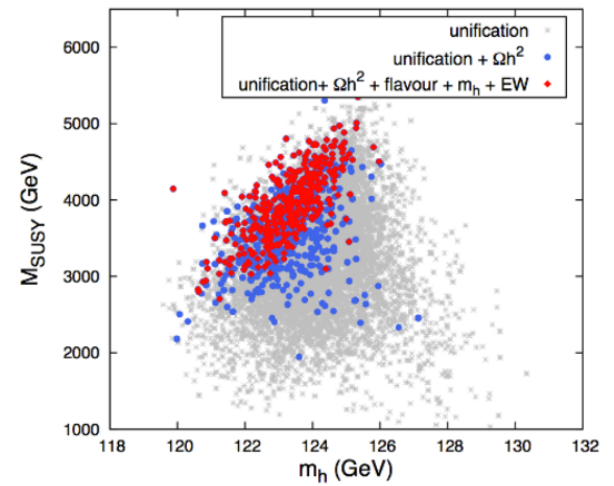

(a)

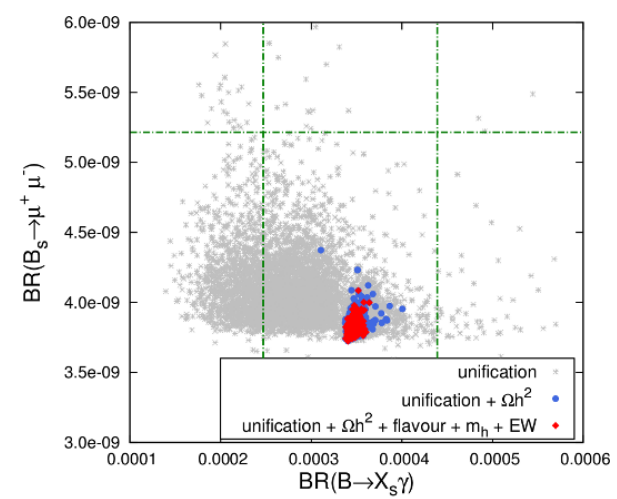

(b)

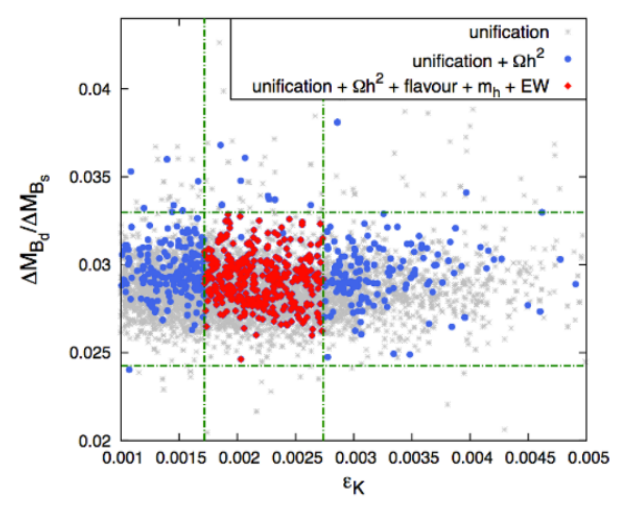

(c)

Figure 6. Scatter plot of the GFV SU(5) points in the planes $\left(m_{h}, M_{\mathrm{SUSY}}\right)(\mathrm{a})$, (BR $\left(\overline{\mathrm{B}} \rightarrow \mathrm{X}_{\mathrm{s}} \gamma\right)$, $\left.\mathrm{BR}\left(\mathrm{B}_{\mathrm{s}} \rightarrow \mu^{+} \mu^{-}\right)\right)$(b), and $\left(\epsilon_{K}, \Delta M_{B_{d}} / \Delta M_{B_{s}}\right)$ (c). Gray stars: all the points that allow the Yukawa coupling unification; blue dots: points satisfying additionally the DM relic density constraint at $3 \sigma$; red diamonds: points with good Yukawa coupling unification and all the constraints listed in table 4 satisfied at $3 \sigma$ (except the LHC). Dashed lines correspond to $3 \sigma$ experimental limits on the corresponding observables.

already being tested by the $\mathrm{LHC} \sqrt{s}=8 \mathrm{TeV}$, as will be discussed in section 4.1.3. It is an interesting aspect of the $\mathrm{SU}(5)$ Yukawa unification scenario that the requirement of satisfying the DM relic density constraint makes it fully testable by the LHC $\sqrt{s}=14 \mathrm{TeV}$ run.

The value of $\tan \beta$ favoured by the DM measurement is also strongly limited, $\tan \beta \in$ $[4,15]$. This is a characteristic feature of the co-annihilation mechanism, as the value of $\tan \beta$ influences the sneutrino mass. It becomes heavier for larger $\tan \beta$, and the efficiency of neutralino-sneutrino co-annihilation drops. What is interesting, however, is that such a limited value of $\tan \beta$ has important consequences for flavour physics observables, as will be discussed in section 4.1.2.

\subsubsection{Higgs, flavour and electroweak observables}

In figure 6 , we present distributions of points for several relevant observables: $\left(m_{h}, M_{\mathrm{SUSY}}\right)$ (a), $\left(\mathrm{BR}\left(\overline{\mathrm{B}} \rightarrow \mathrm{X}_{\mathrm{s}} \gamma\right), \mathrm{BR}\left(\mathrm{B}_{\mathrm{s}} \rightarrow \mu^{+} \mu^{-}\right)\right)(\mathrm{b})$ and $\left(\epsilon_{K}, \Delta M_{B_{d}} / \Delta M_{B_{s}}\right)$ (c). Gray stars indi- 


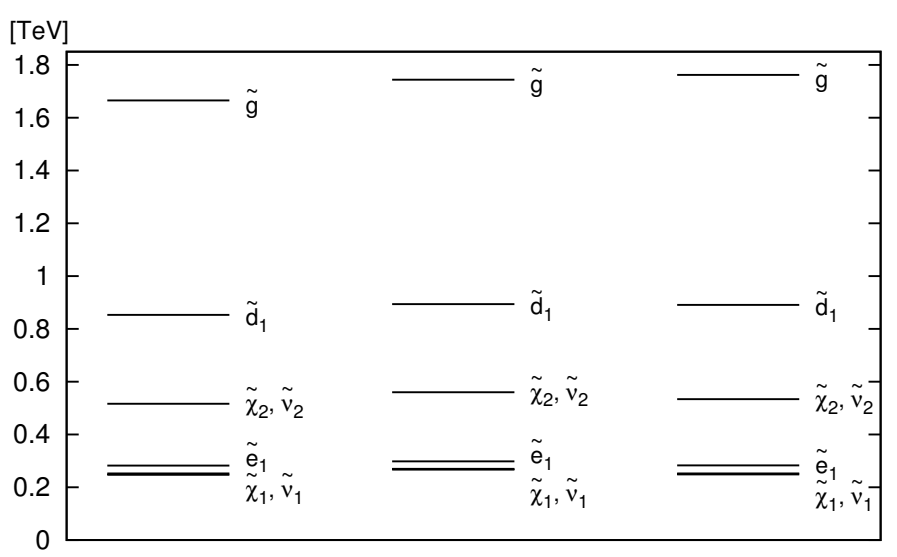

Figure 7. Examples of spectra characteristic of the GFV SU(5) Yukawa unification scenario.

cate all the points for which it is possible to achieve the Yukawa coupling unification for all the three generations. Points that satisfy the relic density constraint at $3 \sigma$ are shown as blue dots, while red diamonds correspond to those cases where additionally all the other constraints listed in table 4 are met at $3 \sigma$ (except the LHC bounds from direct SUSY searches that will be discussed in section 4.1.3).

The Higgs boson mass dependence on the GFV parameters has been discussed in ref. [20-23]. It was shown that while $m_{h}$ can be enhanced by non-zero $(2,3)$ entries of the trilinear down-squark matrix, its dependence on the off-diagonal soft-mass elements is negligible. Therefore, in the scenario considered in this study, the only parameters relevant for the Higgs physics remain $A_{33}^{u}$ and $M_{\text {SUSY }}$. That is confirmed by the panel (a) of figure 6 where no tension between the correct value of the Higgs boson mass and the Yukawa unification constraint (driven by large GFV parameters $m_{23}^{d l}$ and $m_{12}^{d l}$ ) is observed. The EW observables are not affected either because the dominant GFV contribution to $M_{W}$ and $\sin ^{2} \theta_{\text {eff }}$ would be driven by the element $m_{23}^{u e}[19]$.

On the other hand, the presence of sizeable off-diagonal entries in the squark mass matrices might lead to disastrously high SUSY contributions to FCNC processes. It turns out, however, that in the considered scenario most of the flavour constraints are quite easily satisfied for the points that have survived imposing the DM experimental limit. This is mainly due to the fact that the coloured sfermions are relatively heavy in our setup, while $\tan \beta$ needs to be low. Its limited size facilitates the Yukawa coupling unification in the first and second family cases, and allows an efficient neutralino-sneutrino co-annihilation, as discussed in section 4.1.1. In consequence, SUSY contributions to the FCNC processes are suppressed. The only exception is the $\bar{K}^{0}-K^{0}$ meson mixing parameter $\epsilon_{K}$ which provides some constraints on the allowed parameter space (see panel (c) of figure 6). Note also that even if the theoretical uncertainties in the determination of $\Delta M_{B_{d}} / \Delta M_{B_{s}}$ and $\epsilon_{K}$ were further reduced no tension would arise, as the accepted points in figure 6(c) are distributed quite uniformly over the $3 \sigma$ region. 


\subsubsection{LHC direct SUSY searches}

All the points from our scan that demonstrate the full Yukawa matrix unification and satisfy the experimental constraints listed in table 4 share the same characteristic pattern of the light part of the spectrum. Three examples are shown in figure 7 . The Nextto-Lightest SUSY particle (NLSP) is the lightest sneutrino, while one charged slepton, neutralino $\tilde{\chi}_{2}^{0}$ and chargino $\tilde{\chi}_{1}^{ \pm}$are slightly heavier. The presence of light sleptons in the spectrum is very important as it leads to a characteristic 3-lepton signature at the LHC. The next particle on the mass ladder is the lightest down-type squark which is followed by the gluino. All the other coloured particles, the remaining sleptons and heavy Higgses are much heavier and effectively decoupled. A comparison of the production cross-sections reveals that the dominant production channels at the LHC would be direct $\tilde{d}_{1} \tilde{d}_{1}, \tilde{\chi}_{1}^{ \pm} \tilde{\chi}_{2}^{0}$ and $\tilde{\chi}_{1}^{ \pm} \tilde{\chi}_{1}^{ \pm}$production. The cross-section for gluino pair production is one order of magnitude lower, and practically all of the produced gluinos decay via $\tilde{g} \rightarrow \tilde{d}_{1} q$. Therefore, the Yukawa unification scenario is characterized by two distinct LHC signatures: 3 leptons plus missing energy (MET) signature for the electroweakino production, and 0 leptons, jets plus MET signature for the coloured particles production.

The strongest limit on the gluino mass comes from the ATLAS 0 lepton, 2-6 jets plus MET inclusive search [52] that provides a stringent 95\% C.L. exclusion bound $m_{\tilde{g}} \gtrsim$ $1400 \mathrm{GeV}$ for neutralino LSP lighter than $300 \mathrm{GeV}$. The strongest bound on the lightest sbottom mass comes from the CMS 0 leptons, 2 jets and MET search [51], while in the electroweakino sector the most stringent experimental exclusion limits are obtained using the 3-lepton plus MET CMS search [53].

However, one needs to keep in mind that the bounds provided by the experimental collaborations are interpreted in the Simplified Model Scenarios (SMS) that make strong assumptions about the hierarchy of the spectrum and the decay branching ratios. Usually it is assumed that there is only one light SUSY particle apart from the neutralino LSP, and only one decay channel of the NLSP with the branching ratio set to $100 \%$ is considered. In a more general case, however, the presence of other light particles in the spectrum may alter the decay chain, and the assumption concerning the branching ratio may not hold either. In such a case, the exclusion limits for the SMS should be treated with care, and the actual limits are expected to be weaker.

In our analysis, we have used the experimental exclusion bound for the gluino mass [52] at face value because this search is inclusive and therefore tests any gluino-driven multijet signature, irrespectively of a particular decay chain. We have also decided to use a direct 95\% C.L. limit from the CMS sbottom production search [51]. For the SMS T2bb, it reads $m_{\tilde{b}_{1}} \gtrsim 700 \mathrm{GeV}$ for $m_{\tilde{\chi}_{1}^{0}} \simeq 150 \mathrm{GeV}$, and $m_{\tilde{b}_{1}} \gtrsim 640 \mathrm{GeV}$ for $m_{\tilde{\chi}_{1}^{0}} \simeq 250 \mathrm{GeV}$. In our scenario, the sbottom decay corresponds exactly to the SMS T2bb, i.e. $\operatorname{BR}(\tilde{b} \rightarrow$ $\left.b \tilde{\chi}_{1}^{0}\right)=100 \%$. We neglect here the possibility that the actual limit can be weakened due to significant mixing between the sbottoms and other down-type squarks, and we leave a detailed analysis of the GFV effects in such a case for future studies. We will see, however, that this simplifying assumption is justified by the fact that the limits derived from ref. [51] are not the dominant ones. 


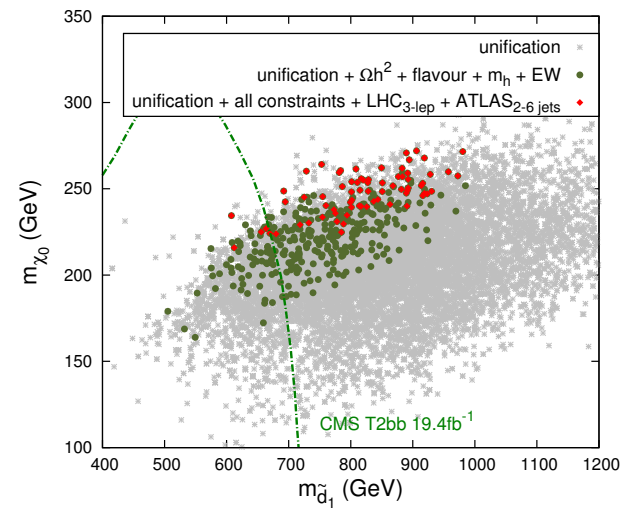

(a)

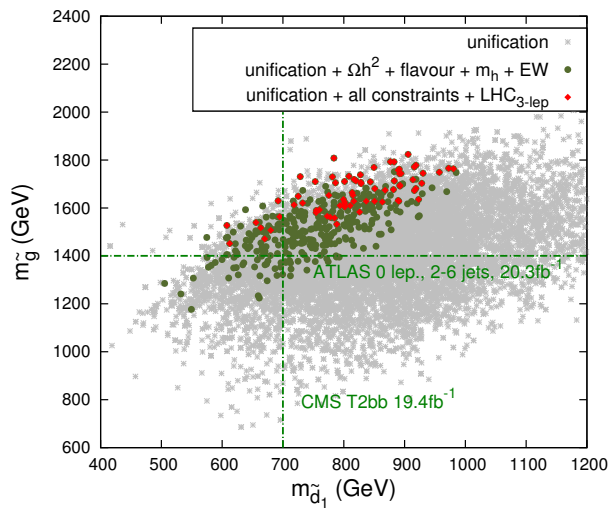

(b)

Figure 8. Scatter plot of the GFV SU(5) points in the planes $\left(m_{\tilde{d}_{1}}, m_{\tilde{\chi}_{1}^{0}}\right)(\mathrm{a})$, and $\left(m_{\tilde{d}_{1}}, m_{\tilde{g}}\right)(\mathrm{b})$. Gray stars: all the points that allow the Yukawa coupling unification; dark green dots: points with good Yukawa coupling unification and all the constraints listed in table 4 (except the LHC) satisfied at $3 \sigma$; red diamonds: points additionally surviving the CMS 3-lepton search at $3 \sigma$. Dashed lines correspond to $95 \%$ C.L. limits provided by other LHC SUSY searches discussed in the text.

On the other hand, the interpretation of the CMS 3-lepton search strongly depends on the hierarchy in the considered spectrum, as well as on actual branching ratios for neutralino and chargino decays. Therefore, in order to correctly quantify the effect of this search in the SU(5) GFV scenario, we perform a full reinterpretation of the experimental analysis using the tools developed first in ref. [24], and modified to recast the limits from SMS in ref. [55]. For the purpose of the present study, we have updated the previously implemented [56] CMS 3 -lepton search to include the full set of data with integrated luminosity of $19.5 / \mathrm{fb}$ [53].

In figure 8 , we present the distribution of the model points in the $\left(m_{\tilde{d}_{1}}, m_{\tilde{\chi}_{1}^{0}}\right)$ plane (a), and in the $\left(m_{\tilde{d}_{1}}, m_{\tilde{g}}\right)$ plane (b). All the points for which Yukawa coupling unification is possible are shown as gray stars, and those that additionally satisfy the experimental constraints listed in table 4 (except the LHC) at $3 \sigma$ are shown as dark green dots. Finally, red diamonds depict the points that survive (at $3 \sigma$ ) the CMS 3-lepton plus MET search. Dashed lines correspond to the 95\% C.L. exclusion bounds from the CMS and ATLAS multijet searches described above, and should be interpreted as lower bounds on sbottom and gluino masses. One can see that already at the LHC $\sqrt{s}=8 \mathrm{TeV}$, the 3-lepton search provides a very strong constraint on the Yukawa unification scenario, much stronger than the limits from refs. [51] and [52]. It is due to the presence of one generation of light sleptons in the spectrum, although the efficiency of the search is weakened with respect to the corresponding SMS, for which the interpretations are provided in the experimental analysis [53].

More importantly, with twice as large a centre-of-mass energy and a luminosity higher by more than an order of magnitude, the 3-lepton search at $\sqrt{s}=14 \mathrm{TeV}$ should easily test spectra with neutralino mass in the ballpark of $250 \mathrm{GeV}$, one slightly heavier generation of sleptons, and the lightest chargino around $500 \mathrm{GeV}$. Therefore, the scenario investigated here is likely to be fully testable in the first months of the LHC $14 \mathrm{TeV}$ run. 


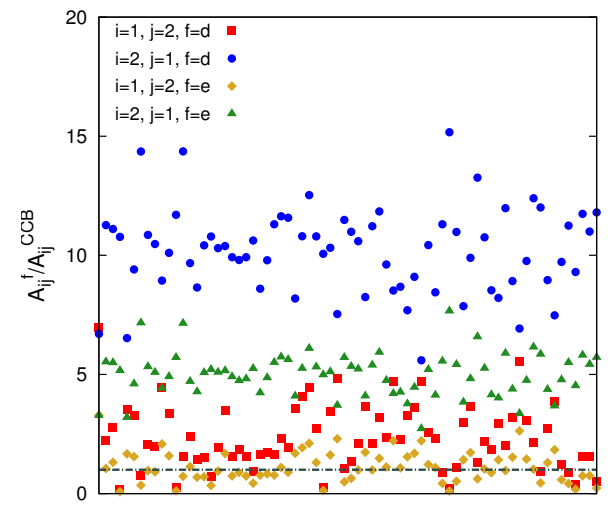

(a)

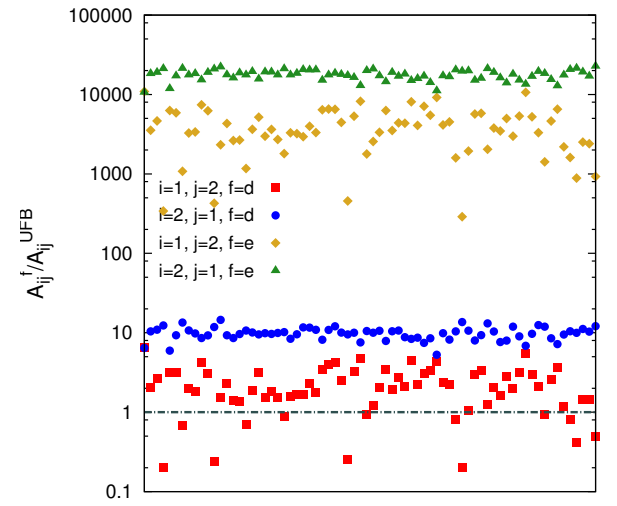

(b)

Figure 9. EW vacuum CCB (a) and UFB (b) stability bounds on the elements $A_{12 / 21}^{d}$ and $A_{12 / 21}^{e}$. Dashed line indicates the upper limit on the allowed size of the off-diagonal trilinear terms.

\subsection{EW vacuum stability}

As discussed in section 3, non-zero elements $A_{12 / 21}^{d e}$ are required to achieve the Yukawa coupling unification of both the first and second family. However, off-diagonal entries of the trilinear couplings (as well as the diagonal ones) are strongly constrained by the requirement of $\mathrm{EW}$ vacuum stability. When the flavour-violating entries are too large, a charge/colour breaking (CCB) minimum may appear in the MSSM scalar potential, and it may become deeper than the standard EW one. The potential may also become unbounded from below (UFB) [57-62]. An important feature of all such constraints is that, unlike the FCNC ones, they do not become weaker when the scale $M_{\mathrm{SUSY}}$ is increased.

In the down-squark sector, tree-level formulae for the CCB bounds are given by [62]

$$
\left(v_{d} / \sqrt{2}\right) A_{i j}^{d} \leq m_{k}^{d}\left[\left(m_{\tilde{q}}^{2}\right)_{i i}+\left(m_{\tilde{d}}^{2}\right)_{j j}+m_{H_{d}}^{2}+\mu^{2}\right]^{1 / 2}, \quad k=\operatorname{Max}(i, j) .
$$

The limits on $A_{i j}^{e}$ have an analogous form, up to replacing the matrix $m_{\tilde{d}}^{2}$ by $m_{\tilde{e}}^{2}$. Similarly, the UFB bounds read [62]

$$
\begin{aligned}
& \left(v_{d} / \sqrt{2}\right) A_{i j}^{d} \leq m_{k}^{d}\left[\left(m_{\tilde{q}}^{2}\right)_{i i}+\left(m_{\tilde{d}}^{2}\right)_{j j}+\left(m_{\tilde{l}}^{2}\right)_{i i}+\left(m_{\tilde{e}}^{2}\right)_{j j}\right]^{1 / 2} \\
& \left(v_{d} / \sqrt{2}\right) A_{i j}^{e} \leq \sqrt{3} m_{k}^{l}, \quad k=\operatorname{Max}(i, j) .
\end{aligned}
$$

In figure 9, we show to what extent the CCB and UFB limits are satisfied for the points that allow Yukawa coupling unification and satisfy at $3 \sigma$ all the experimental constraints listed in table 4. Dashed line indicates the upper limit on the allowed size of the off-diagonal trilinear terms. One can see that the CCB stability bounds are violated by around an order of magnitude. The situation is even worse in the case of the UFB bounds where the size of the elements $A_{i j}^{e}$ are around four orders of magnitude larger than it is allowed by the stability constraint. It results from the fact that the UFB limit on $A_{i j}^{e}$ is of the order of the muon mass. Therefore, we conclude that the EW MSSM vacuum is not stable in the Yukawa unification scenario considered in our study 
On the other hand, the appearance of a CCB minimum does not necessarily exclude the considered model, as long as the standard EW vacuum lives longer than the age of the universe. Moreover, in such a case, the UFB bound becomes irrelevant because the probability of a tunnelling process along the CCB direction is much higher [63]. To derive metastability bounds, the bounce action for a given scalar potential should be calculated numerically, which is beyond the scope of our paper. To evaluate the impact of metastability on the validity of the unification scenario, we will use instead the results of the analysis performed in ref. [63]. The derived metastability bounds do not depend on the Yukawa couplings, and therefore are in general much less stringent than the CCB ones. The effect is the strongest for the $A_{12 / 21}$ elements, in which case the CCB limit can be weakened by three to four orders of magnitude, depending on a particular choice of the model parameters. We can therefore conclude that the scenario considered in our study leads to an unstable, but a long-lived vacuum.

It should be stressed, though, that the tension between the Yukawa unification condition and the EW vacuum stability is less severe in our present scenario than in the case of Yukawa unification through large diagonal $A$-terms where the CCB bounds were violated by two orders of magnitude [10]. On the other hand, theoretical calculations of the stability conditions are still marred by many uncertainties. This leaves a possibility that future improvements might further reduce (or even eliminate) the tension between the vacuum stability and the Yukawa unification.

\subsection{Examples of points with successful SU(5) Yukawa matrix unification}

We conclude this section with three examples of benchmark points that satisfy both the requirement of Yukawa coupling unification at the GUT scale, as well as the experimental constraints listed in table 4 . The corresponding input parameters, mass spectra and values of the relevant observables are listed in table 5 .

\section{Conclusions}

In this study, we provided evidence that the SU(5) boundary conditions for the Yukawa matrices at the GUT-scale can be satisfied within the renormalizable $R$-parity-conserving MSSM if a more general flavour structure of the soft SUSY-breaking sector is allowed. In particular, we found that in our scenario a non-zero $(2,3)$ element of the down-type squark mass matrix is crucial to achieve an approximate Yukawa unification for the second family, as the SUSY threshold correction driven by this entry is proportional to the large trilinear coupling $A_{33}^{d e}$. Moreover, simultaneous unification of the Yukawa couplings for the second and first family additionally requires the elements $m_{12}^{d l}, m_{13}^{d l}$ and $A_{12 / 21}^{d e}$ to assume non-zero values. The latter, however, can be kept relatively small, which helps to ease the tension between the EW vacuum stability and the Yukawa unification with respect to the scenario relying on large diagonal $A$-terms.

The FCNC processes are often raised as an argument against the GFV MSSM. We can observe that, to the contrary, the Yukawa unification scenario considered in this study is consistent with a wide set of experimental measurements, including the FCNC processes. 


\begin{tabular}{|c|c|c|c|}
\hline & $\mathrm{P} 1$ & $\mathrm{P} 2$ & P3 \\
\hline$M_{1 / 2}$ & 643 & 653 & 666 \\
\hline$m_{H_{u}}, m_{H_{d}}$ & 1745,1551 & 1856,1781 & 2770,1550 \\
\hline $\tan \beta$ & 9.5 & 8.3 & 8.4 \\
\hline$A_{33}^{d e}, A_{33}^{u}$ & 913,1561 & $741,-4870$ & 841,425 \\
\hline$A_{11}^{d e} / A_{33}^{d e}$ & -0.00013 & -0.00003 & 0.00012 \\
\hline$A_{22}^{d e} / A_{33}^{d e}$ & 0.058 & 0.042 & 0.055 \\
\hline$A_{12}^{d e} / A_{33}^{d e}, A_{21}^{d e} / A_{33}^{d e}$ & $0.017,0.143$ & $0.007,0.167$ & $0.074,0.073$ \\
\hline$A_{22}^{u} / A_{33}^{u}$ & -0.003 & 0.004 & -0.0005 \\
\hline$m_{11}^{d l}, m_{22}^{d l}, m_{33}^{d l}$ & $1485,1315,1755$ & $1734,1156,1896$ & $1578,1219,1925$ \\
\hline$m_{23}^{d l} / m_{33}^{d l}$ & 0.81 & 0.72 & 0.77 \\
\hline$m_{13}^{d l} / m_{33}^{d l}$ & 0.23 & 0.23 & 0.29 \\
\hline$m_{12}^{d l} / m_{33}^{d l}$ & 0.43 & 0.43 & 0.44 \\
\hline$m_{11}^{u e}, m_{22}^{u e}, m_{33}^{u e}$ & $3511,3540,4592$ & $5264,5309,6240$ & $4004,4038,5179$ \\
\hline$Y_{d}, Y_{e}$ at $M_{\mathrm{GUT}}$ & $0.000019,0.000018$ & $0.000018,0.000016$ & $0.000013,0.000015$ \\
\hline$Y_{s}, Y_{\mu}$ at $M_{\mathrm{GUT}}$ & $0.0036,0.0037$ & $0.0030,0.0033$ & $0.0028,0.0032$ \\
\hline$Y_{b}, Y_{\tau}$ at $M_{\mathrm{GUT}}$ & $0.067,0.062$ & $0.058,0.055$ & $0.054,0.054$ \\
\hline$\mu$ & -3862 & -6142 & -3856 \\
\hline$m_{A}, m_{H^{ \pm}}$ & 3603,3570 & 6242,6161 & 3847,3809 \\
\hline$m_{h}$ & 123.3 & 126.0 & 124.5 \\
\hline$m_{\tilde{g}}$ & 1665 & 1744 & 1762 \\
\hline$m_{\tilde{\chi}_{1}^{0}}, m_{\tilde{\chi}_{2}^{0}}$ & 247,516 & 267,560 & 249,534 \\
\hline$m_{\chi_{3}^{0}}, m_{\chi_{4}^{0}}$ & 3684,3684 & 6110,6111 & 3862,3862 \\
\hline$m_{\tilde{\chi}_{1}^{ \pm}}, m_{\chi_{2}^{ \pm}}$ & 516,3685 & 560,6111 & 534,3863 \\
\hline$m_{\tilde{d}_{1}}, m_{\tilde{d}_{2}}, m_{\tilde{d}_{3}}$ & $853,1838,2245$ & $894,1935,2238$ & $891,1889,2364$ \\
\hline$m_{\tilde{d}_{4}}, m_{\tilde{d}_{5}}, m_{\tilde{d}_{6}}$ & $3720,3750,4057$ & $5162,5399,5449$ & $4200,4231,4525$ \\
\hline$m_{\tilde{u}_{1}}, m_{\tilde{u}_{2}}, m_{\tilde{u}_{3}}$ & $3457,3711,3719$ & $3757,5165,5390$ & $3776,4200,4216$ \\
\hline$m_{\tilde{u}_{4}}, m_{\tilde{u}_{5}}, m_{\tilde{u}_{6}}$ & $3740,3749,4059$ & $5399,5435,5449$ & $4231,4249,4527$ \\
\hline$m_{\tilde{e}_{1}}, m_{\tilde{e}_{2}}, m_{\tilde{e}_{3}}$ & $282,1490,2097$ & $298,1691,2116$ & $283,1598,2253$ \\
\hline$m_{\tilde{e}_{4}}, m_{\tilde{e}_{5}}, m_{\tilde{e}_{6}}$ & $3512,3538,4464$ & $5267,5310,6176$ & $3973,4007,5052$ \\
\hline$m_{\tilde{\nu}_{1}}, m_{\tilde{\nu}_{2}}, m_{\tilde{\nu}_{3}}$ & $252,1488,2098$ & $270,1690,2117$ & $252,1596,2254$ \\
\hline$\chi_{\mathrm{LHC}}^{2}$ & 5.1 & 9.2 & 11.2 \\
\hline$\Omega_{\chi} h^{2}$ & 0.141 & 0.112 & 0.088 \\
\hline$\sigma_{p}^{\mathrm{SI}}$ & $5.0 \times 10^{-13}$ & $2.7 \times 10^{-13}$ & $6.0 \times 10^{-13}$ \\
\hline $\mathrm{BR}\left(\overline{\mathrm{B}} \rightarrow \mathrm{X}_{\mathrm{s}} \gamma\right)$ & $3.47 \times 10^{-4}$ & $3.42 \times 10^{-4}$ & $3.44 \times 10^{-4}$ \\
\hline $\mathrm{BR}\left(\mathrm{B}_{\mathrm{s}} \rightarrow \mu^{+} \mu^{-}\right)$ & $3.92 \times 10^{-9}$ & $3.82 \times 10^{-9}$ & $3.84 \times 10^{-9}$ \\
\hline $\mathrm{BR}\left(\mathrm{B}_{\mathrm{d}} \rightarrow \mu^{+} \mu^{-}\right)$ & $1.18 \times 10^{-10}$ & $1.20 \times 10^{-10}$ & $1.17 \times 10^{-10}$ \\
\hline$\Delta M_{B_{s}}$ & $1.12 \times 10^{-11}$ & $1.08 \times 10^{-11}$ & $1.09 \times 10^{-11}$ \\
\hline$\Delta M_{B_{d}}$ & $3.29 \times 10^{-13}$ & $2.92 \times 10^{-13}$ & $3.29 \times 10^{-13}$ \\
\hline$\Delta M_{B_{d}} / \Delta M_{B_{d}}$ & 0.029 & 0.030 & 0.031 \\
\hline $\sin (2 \beta)$ & 0.732 & 0.689 & 0.649 \\
\hline $\mathrm{BR}\left(\mathrm{B}_{\mathrm{u}} \rightarrow \tau \nu\right)$ & $7.52 \times 10^{-5}$ & $7.53 \times 10^{-5}$ & $7.53 \times 10^{-5}$ \\
\hline$\epsilon_{K}$ & 0.0025 & 0.0018 & 0.0021 \\
\hline $\mathrm{BR}\left(K^{+} \rightarrow \pi^{+} \nu \bar{\nu}\right)$ & $8.37 \times 10^{-11}$ & $8.39 \times 10^{-11}$ & $8.42 \times 10^{-11}$ \\
\hline$\left|d_{n}\right|$ & $1.4 \times 10^{-29}$ & $5.3 \times 10^{-28}$ & $2.1 \times 10^{-28}$ \\
\hline $\sin ^{2} \theta_{\text {eff }}$ & 0.2314 & 0.2314 & 0.2314 \\
\hline$M_{W}$ & 80.377 & 80.374 & 80.380 \\
\hline
\end{tabular}

Table 5. Benchmark points for the Yukawa unification GFV SU(5) scenario. 
The consistency would remain true even when theoretical uncertainties in determination of the flavour observables were reduced. We showed that a non-trivial flavour structure of the down-squark sector does not pose a threat for the flavour observables for two reasons. First, the value of $\tan \beta$ required by both the Yukawa unification condition and the DM relic density measurement must stay in the range of $\tan \beta \in[5,15]$, so no large $\tan \beta$ enhancements occurs in supersymmetric loop corrections to rare $B$-meson decays. Secondly, squarks remain relatively heavy. This may suggest that the principle of Minimal Flavour Violation as a unique way to remain in agreement with the flavour observables is not always well motivated.

Another interesting feature of the GFV SU(5) unification scenario are the properties of the dark matter. It turns out that the condition for Yukawa matrix unification strictly requires an almost purely bino-like neutralino LSP with the mass in the ballpark of $250 \mathrm{GeV}$. This, in turn, enforces a particular hierarchy of masses in the corresponding SUSY spectrum, with sneutrino NLSP and one light charged slepton. Spectra of this kind, that lead to a characteristic 3-lepton collider signature, have started to be tested in the direct SUSY searches at the LHC $\sqrt{s}=8 \mathrm{TeV}$. In the upcoming LHC Run II, they are going to be tested in a complete manner.

Let us conclude with one more remark. The aim of our study was to check whether large GUT-scale threshold corrections to the Yukawa couplings or modifications of the Yukawa boundary conditions can be avoided in the $R$-parity conserving MSSM. We did not try, however, to construct a full and self-consistent model valid above the GUT-scale. For this reason some issues related to the minimal SU(5) GUT, like the proton lifetime, remained unaddressed. On the other hand, ways of saving the proton from decaying too fast in the framework of minimal $\mathrm{SU}(5)$ have been proposed in the literature, for example by employing higher-dimensional operators [3]. It seems, therefore, feasible to combine two complementary approaches to the Yukawa coupling unification, the one using flavourviolating low-scale threshold corrections, and the one introducing higher-dimensional operators. A quantitatively correct SU(5) UV-completion of the MSSM could be constructed this way.

Note added in proof: after the review process of this article had been completed, we realized that the limits from Lepton Flavour Violating (LFV) processes, in particular from $\mathrm{BR}\left(\mu^{+} \rightarrow e^{+} \gamma\right)$ as measured by the MEG Collaboration [64], provide a complementary way to experimentally test our scenario.

To enhance threshold corrections to the down-quark Yukawa couplings, we have used significant off-diagonal entries in the down-squark soft mass matrices. The $S U(5)$ GUT relations connect them with the slepton soft mass matrices. In this way, a sizeable mixing between sleptons of the first and second family is generated. We checked that, unfortunately, all the model points that satisfy the phenomenological constraints discussed in section 4 produce a branching ratio above the $90 \%$ C.L. upper bound reported in ref. [64]. On the other hand, experimental limits on $\operatorname{BR}\left(\tau^{+} \rightarrow \mu^{+} \gamma\right)$ are not violated.

There are several ways to render our scenario consistent with the MEG bound. One of them is abandoning the GUT relations between the squark and slepton soft terms, while 
still insisting on the full Yukawa matrix unification. The other is to invoke alternative mechanisms of Yukawa matrix unification in the first generation case, in which the necessary effects are small, and could easily be attributed, e.g., to higher-dimensional operators at the GUT scale. In such an approach, the (12) and (13) entries of the down-squark mass matrix could be set to zero, while the non-zero (23) entry could remain large enough for the (more difficult) second-generation Yukawa unification, without violating the $\operatorname{BR}\left(\mu^{+} \rightarrow e^{+} \gamma\right)$ and $\mathrm{BR}\left(\tau^{+} \rightarrow \mu^{+} \gamma\right)$ bounds.

\section{Acknowledgments}

We would like to thank Werner Porod for great help with issues related to SPheno. We also would like to thank Andreas Crivellin, Christophe Grojean, Gian Giudice, Mikołaj Misiak, Ulrich Nierste and Enrico Maria Sessolo for many useful comments and discussions. M.I. was supported in part by the Foundation for Polish Science International PhD Projects Programme co-financed by the EU European Regional Development Fund, by the Karlsruhe Institute of Technology, and by the National Science Centre (Poland) research project, decision DEC-2014/13/B/ST2/03969. K.K. was supported by the EU and MSHE Grant No. POIG.02.03.00-00-013/09. The use of the CIS computer cluster at the National Centre for Nuclear Research is gratefully acknowledged.

Open Access. This article is distributed under the terms of the Creative Commons Attribution License (CC-BY 4.0), which permits any use, distribution and reproduction in any medium, provided the original author(s) and source are credited.

\section{References}

[1] S. Dimopoulos and H. Georgi, Softly broken supersymmetry and SU(5), Nucl. Phys. B 193 (1981) 150 [INSPIRE].

[2] H. Georgi and C. Jarlskog, A new lepton - Quark mass relation in a unified theory, Phys. Lett. B 86 (1979) 297 [INSPIRE].

[3] D. Emmanuel-Costa and S. Wiesenfeldt, Proton decay in a consistent supersymmetric SU(5) GUT model, Nucl. Phys. B 661 (2003) 62 [hep-ph/0302272] [INSPIRE].

[4] S. Antusch and M. Spinrath, New GUT predictions for quark and lepton mass ratios confronted with phenomenology, Phys. Rev. D 79 (2009) 095004 [arXiv:0902.4644] [INSPIRE].

[5] S. Antusch, S.F. King and M. Spinrath, GUT predictions for quark-lepton Yukawa coupling ratios with messenger masses from non-singlets, Phys. Rev. D 89 (2014) 055027 [arXiv: 1311.0877] [INSPIRE].

[6] W. Buchmüller and D. Wyler, $C P$ violation and $R$ invariance in supersymmetric models of strong and electroweak interactions, Phys. Lett. B 121 (1983) 321 [INSPIRE].

[7] L.J. Hall, V.A. Kostelecky and S. Raby, New flavor violations in supergravity models, Nucl. Phys. B 267 (1986) 415 [INSPIRE].

[8] J.L. Diaz-Cruz, H. Murayama and A. Pierce, Can supersymmetric loops correct the fermion mass relations in SU(5)?, Phys. Rev. D 65 (2002) 075011 [hep-ph/0012275] [INSPIRE]. 
[9] T. Enkhbat, SU(5) unification for Yukawas through SUSY threshold effects, arXiv:0909.5597 [INSPIRE].

[10] M. Iskrzynski, Effects of supersymmetric threshold corrections on the Yukawa matrix unification, Eur. Phys. J. C 75 (2015) 51 [arXiv:1408.2165] [InSPIRE].

[11] A. Crivellin, L. Hofer and J. Rosiek, Complete resummation of chirally-enhanced loop-effects in the MSSM with non-minimal sources of flavor-violation, JHEP 07 (2011) 017 [arXiv:1103.4272] [INSPIRE].

[12] A. Crivellin and U. Nierste, Supersymmetric renormalisation of the CKM matrix and new constraints on the squark mass matrices, Phys. Rev. D 79 (2009) 035018 [arXiv:0810.1613] [INSPIRE].

[13] A. Crivellin, Effective Higgs vertices in the generic MSSM, Phys. Rev. D 83 (2011) 056001 [arXiv: 1012.4840] [INSPIRE].

[14] A. Crivellin and J. Girrbach, Constraining the MSSM sfermion mass matrices with light fermion masses, Phys. Rev. D 81 (2010) 076001 [arXiv: 1002.0227] [INSPIRE].

[15] J. Guasch and J. Solà, FCNC top quark decays: a door to SUSY physics in high luminosity colliders?, Nucl. Phys. B 562 (1999) 3 [hep-ph/9906268] [INSPIRE].

[16] J.J. Cao et al., SUSY-induced FCNC top-quark processes at the large hadron collider, Phys. Rev. D 75 (2007) 075021 [hep-ph/0702264] [INSPIRE].

[17] S. Fichet, B. Herrmann and Y. Stoll, A new flavour imprint of SU(5)-like grand unification and its LHC signatures, Phys. Lett. B 742 (2015) 69 [arXiv:1403.3397] [INSPIRE].

[18] B. Herrmann, M. Klasen and Q. Le Boulc'h, Impact of squark flavour violation on neutralino dark matter, Phys. Rev. D 84 (2011) 095007 [arXiv: 1106.6229] [InSPIRE].

[19] S. Heinemeyer, W. Hollik, F. Merz and S. Penaranda, Electroweak precision observables in the MSSM with nonminimal flavor violation, Eur. Phys. J. C 37 (2004) 481 [hep-ph/0403228] [INSPIRE].

[20] J. Cao, G. Eilam, K.-i. Hikasa and J.M. Yang, Experimental constraints on stop-scharm flavor mixing and implications in top-quark FCNC processes, Phys. Rev. D 74 (2006) 031701 [hep-ph/0604163] [INSPIRE].

[21] M. Arana-Catania, S. Heinemeyer, M.J. Herrero and S. Penaranda, Higgs boson masses and B-physics constraints in non-minimal flavor violating SUSY scenarios, JHEP 05 (2012) 015 [arXiv: 1109.6232] [INSPIRE].

[22] M. Arana-Catania, S. Heinemeyer and M.J. Herrero, Updated constraints on general squark flavor mixing, Phys. Rev. D 90 (2014) 075003 [arXiv:1405.6960] [INSPIRE].

[23] K. Kowalska, Phenomenology of SUSY with general flavour violation, JHEP 09 (2014) 139 [arXiv: 1406.0710] [INSPIRE].

[24] A. Fowlie et al., The CMSSM favoring new territories: the impact of new LHC limits and a 125 GeV Higgs, Phys. Rev. D 86 (2012) 075010 [arXiv: 1206. 0264] [INSPIRE].

[25] F. Feroz, M.P. Hobson and M. Bridges, MultiNest: an efficient and robust Bayesian inference tool for cosmology and particle physics, Mon. Not. Roy. Astron. Soc. 398 (2009) 1601 [arXiv: 0809.3437] [INSPIRE].

[26] W. Porod and F. Staub, SPheno 3.1: extensions including flavour, CP-phases and models beyond the MSSM, Comput. Phys. Commun. 183 (2012) 2458 [arXiv:1104.1573] [InSPIRE].

[27] Particle Data Group collaboration, K.A. Olive et al., Review of particle physics, Chin. Phys. C 38 (2014) 090001 [INSPIRE]. 
[28] http://www.utfit.org/UTfit/ResultsSummer2014PostMoriondNP.

[29] A. Crivellin et al., SUSY_FLAVOR v2: a computational tool for FCNC and CP-violating processes in the MSSM, Comput. Phys. Commun. 184 (2013) 1004 [arXiv:1203.5023] [INSPIRE].

[30] S. Aoki et al., Review of lattice results concerning low-energy particle physics, Eur. Phys. J. C 74 (2014) 2890 [arXiv:1310.8555] [INSPIRE].

[31] Heavy Flavor Averaging Group collaboration, Y. Amhis et al., Averages of b-hadron, c-hadron and $\tau$-lepton properties as of summer 2014, arXiv:1412.7515 [INSPIRE].

[32] M. Misiak et al., Estimate of $B\left(\bar{B} \rightarrow X_{s} \gamma\right)$ at $O\left(\alpha_{s}^{2}\right)$, Phys. Rev. Lett. 98 (2007) 022002 [hep-ph/0609232] [INSPIRE].

[33] C. Bobeth et al., $B_{s, d} \rightarrow \ell^{+} \ell^{-}$in the standard model with reduced theoretical uncertainty, Phys. Rev. Lett. 112 (2014) 101801 [arXiv:1311.0903] [INSPIRE].

[34] J. Brod and M. Gorbahn, Next-to-next-to-leading-order charm-quark contribution to the cp-violation parameter $\epsilon_{K}$ and $\Delta M_{K}$, Phys. Rev. Lett. 108 (2012) 121801 [arXiv: 1108.2036] [INSPIRE].

[35] P. Gondolo et al., DarkSUSY: computing supersymmetric dark matter properties numerically, JCAP 07 (2004) 008 [astro-ph/0406204] [INSPIRE].

[36] T. Hahn, S. Heinemeyer, W. Hollik, H. Rzehak and G. Weiglein, High-precision predictions for the light CP-even Higgs boson mass of the minimal supersymmetric standard model, Phys. Rev. Lett. 112 (2014) 141801 [arXiv:1312.4937] [INSPIRE].

[37] M. Frank et al., The Higgs boson masses and mixings of the complex MSSM in the Feynman-diagrammatic approach, JHEP 02 (2007) 047 [hep-ph/0611326] [INSPIRE].

[38] G. Degrassi, S. Heinemeyer, W. Hollik, P. Slavich and G. Weiglein, Towards high precision predictions for the MSSM Higgs sector, Eur. Phys. J. C 28 (2003) 133 [hep-ph/0212020] [INSPIRE].

[39] S. Heinemeyer, W. Hollik and G. Weiglein, FeynHiggs: a program for the calculation of the masses of the neutral CP even Higgs bosons in the MSSM, Comput. Phys. Commun. 124 (2000) 76 [hep-ph/9812320] [INSPIRE].

[40] P. Bechtle, O. Brein, S. Heinemeyer, G. Weiglein and K.E. Williams, HiggsBounds: confronting arbitrary Higgs sectors with exclusion bounds from LEP and the Tevatron, Comput. Phys. Commun. 181 (2010) 138 [arXiv:0811.4169] [INSPIRE].

[41] P. Bechtle, O. Brein, S. Heinemeyer, G. Weiglein and K.E. Williams, HiggsBounds 2.0.0: confronting neutral and charged Higgs sector predictions with exclusion bounds from LEP and the Tevatron, Comput. Phys. Commun. 182 (2011) 2605 [arXiv:1102.1898] [INSPIRE].

[42] P. Bechtle et al., HiggsBounds-4: improved tests of extended Higgs sectors against exclusion bounds from LEP, the Tevatron and the LHC, Eur. Phys. J. C 74 (2014) 2693 [arXiv: 1311.0055] [INSPIRE].

[43] P. Bechtle et al., HiggsSignals: confronting arbitrary Higgs sectors with measurements at the Tevatron and the LHC, Eur. Phys. J. C 74 (2014) 2711 [arXiv:1305.1933] [InSPIRE].

[44] LUX collaboration, D.S. Akerib et al., First results from the LUX dark matter experiment at the Sanford Underground Research Facility, Phys. Rev. Lett. 112 (2014) 091303 [arXiv: 1310.8214] [INSPIRE].

[45] K. Cheung et al., Global study of the simplest scalar phantom dark matter model, JCAP 10 (2012) 042 [arXiv:1207.4930] [INSPIRE]. 
[46] K. Kowalska, L. Roszkowski, E.M. Sessolo and S. Trojanowski, Low fine tuning in the MSSM with higgsino dark matter and unification constraints, JHEP 04 (2014) 166 [arXiv: 1402.1328] [INSPIRE].

[47] Planck collaboration, P.A.R. Ade et al., Planck 2013 results. XVI. Cosmological parameters, Astron. Astrophys. 571 (2014) A16 [arXiv:1303.5076] [INSPIRE].

[48] CMS collaboration, Combination of standard model Higgs boson searches and measurements of the properties of the new boson with a mass near $125 \mathrm{GeV}$, CMS-PAS-HIG-13-005 (2013).

[49] CMS, LHCB collaboration, V. Khachatryan et al., Observation of the rare $B_{s}^{0} \rightarrow \mu^{+} \mu^{-}$ decay from the combined analysis of CMS and LHCb data, arXiv:1411.4413 [INSPIRE].

[50] C.A. Baker et al., An improved experimental limit on the electric dipole moment of the neutron, Phys. Rev. Lett. 97 (2006) 131801 [hep-ex/0602020] [INSPIRE].

[51] CMS Collaboration, Search for direct production of bottom squark pairs, CMS-PAS-SUS-13-018 (2013).

[52] ATLAS collaboration, Search for squarks and gluinos with the ATLAS detector in final states with jets and missing transverse momentum using $\sqrt{s}=8 \mathrm{TeV}$ proton-proton collision data, JHEP 09 (2014) 176 [arXiv:1405.7875] [INSPIRE].

[53] ATLAS collaboration, Search for direct production of charginos, neutralinos and sleptons in final states with two leptons and missing transverse momentum in pp collisions at $\sqrt{s}=8 \mathrm{TeV}$ with the ATLAS detector, JHEP 05 (2014) 071 [arXiv:1403.5294] [INSPIRE].

[54] XENON1T collaboration, E. Aprile, The XENON1T dark matter search experiment, Springer Proc. Phys. C12-02-22 (2013) 93 [arXiv: 1206.6288] [INSPIRE].

[55] K. Kowalska and E.M. Sessolo, Natural MSSM after the LHC 8 TeV run, Phys. Rev. D 88 (2013) 075001 [arXiv:1307.5790] [INSPIRE].

[56] CMS collaboration, Search for direct EWK production of SUSY particles in multilepton modes with 8TeV data, CMS-PAS-SUS-12-022 (2012).

[57] J.M. Frere, D.R.T. Jones and S. Raby, Fermion masses and induction of the weak scale by supergravity, Nucl. Phys. B 222 (1983) 11 [INSPIRE].

[58] L. Álvarez-Gaumé, J. Polchinski and M.B. Wise, Minimal low-energy supergravity, Nucl. Phys. B 221 (1983) 495 [INSPIRE].

[59] J.P. Derendinger and C.A. Savoy, Quantum effects and $\mathrm{SU}(2) \times \mathrm{U}(1)$ breaking in supergravity gauge theories, Nucl. Phys. B 237 (1984) 307 [InSPIRE].

[60] C. Kounnas, A.B. Lahanas, D.V. Nanopoulos and M. Quirós, Low-energy behavior of realistic locally supersymmetric grand unified theories, Nucl. Phys. B 236 (1984) 438 [INSPIRE].

[61] J.A. Casas, A. Lleyda and C. Muñoz, Strong constraints on the parameter space of the MSSM from charge and color breaking minima, Nucl. Phys. B 471 (1996) 3 [hep-ph/9507294] [INSPIRE].

[62] J.A. Casas and S. Dimopoulos, Stability bounds on flavor violating trilinear soft terms in the MSSM, Phys. Lett. B 387 (1996) 107 [hep-ph/9606237] [INSPIRE].

[63] J.-H. Park, Metastability bounds on flavour-violating trilinear soft terms in the MSSM, Phys. Rev. D 83 (2011) 055015 [arXiv: 1011.4939] [INSPIRE].

[64] MEG collaboration, J. Adam et al., New constraint on the existence of the $\mu^{+} \rightarrow e^{+} \gamma$ decay, Phys. Rev. Lett. 110 (2013) 201801 [arXiv: 1303. 0754] [INSPIRE]. 